OPEN ACCESS

Edited by:

Alexandre Corthay

Oslo University Hospital, Norway

Reviewed by:

Maria Rosaria Coscia Istituto di Biochimica delle Proteine

(IBP), Italy

Rowena Hoare, University of Stirling, United Kingdom

Lora Petrie-Hanson,

Mississippi State University, United States

${ }^{*}$ Correspondence: Shi Xi Chen chenshixi@xmu.edu.cn

Specialty section This article was submitted to Molecular Innate Immunity, a section of the journal

Frontiers in Immunology

Received: 02 December 2018 Accepted: 11 February 2019

Published: 28 February 2019

Citation:

Qiu HT, Fernandes JMO, Hong WS, Wu HX, Zhang YT, Huang S, Liu DT, Yu H, Wang Q, You XX and Chen SX (2019) Paralogues From the Expanded

TIr11 Gene Family in Mudskipper (Boleophthalmus pectinirostris) Are Under Positive Selection and Respond Differently to LPS/poly(l:C) Challenge.

Front. Immunol. 10:343. doi: 10.3389/fimmu.2019.00343

\section{Paralogues From the Expanded TIr11 Gene Family in Mudskipper (Boleophthalmus pectinirostris) Are Under Positive Selection and Respond Differently to LPS/Poly(I:C) Challenge}

Heng Tong Qiu' ${ }^{1}$, Jorge M. O. Fernandes ${ }^{2}$, Wan Shu Hong ${ }^{1,3}$, Hai Xu Wu ${ }^{4}$, Yu Ting Zhang ${ }^{1}$, Sheng Huang ${ }^{1}$, Dong Teng Liu ${ }^{1}$, Hui Yu ${ }^{4}$, Qiong Wang ${ }^{1}$, Xin Xin You ${ }^{4}$ and Shi Xi Chen ${ }^{1,3,5 *}$

${ }^{1}$ State Key Laboratory of Marine Environmental Science, College of Ocean and Earth Sciences, Xiamen University, Xiamen, China, ${ }^{2}$ Faculty of Biosciences and Aquaculture, Nord University, Bodø, Norway, ${ }^{3}$ Fujian Collaborative Innovation Center for Exploitation and Utilization of Marine Biological Resources, Xiamen, China, ${ }^{4}$ Shenzhen Key Laboratory of Marine Genomics, Marine and Fisheries Institute, BGI-Shenzhen, Shenzhen, China, ${ }^{5}$ State-Province Joint Engineering Laboratory of Marine

Bioproducts and Technology, Xiamen University, Xiamen, China

Toll-like receptors (TLRs) are major molecular pattern recognition receptors, which are essential for triggering a series of innate immune responses against invading pathogens by recognizing their evolutionary conserved molecular patterns. The mudskipper, Boleophthalmus pectinirostris is exceptional among fishes due to its amphibious lifestyle and adaptation to living on mudflats. The whole-genome sequencing of $B$. pectinirostris has revealed that this species possesses an expansion of TIr11 family [12 TIr11 family genes (one t/r21, 4 t/r22, and 7 t/r23)] that we focused on in the present study. The full-length cDNA sequences of the $12 \mathrm{t}$ /rs in $B$. pectinirostris were cloned and their deduced amino acid sequences possessed a typical TLR domain arrangement. Likelihood tests of selection revealed that these 12 Tlr11 family genes are under diversifying selection. A total of 13 sites were found to be positively selected by more than one evolution model, of which 11 were located in the ligand-binding ectodomain. The observed non-synonymous substitutions may have functional implications in antigen and pathogen recognition specificity. These 12 tirs were highly expressed in immune-related tissues, i.e. spleen and kidney. T/r21 and t/r22b transcripts were significantly up-regulated by LPS, whereas t/r22a, t/r22d, t/r23b, t/r23e, t/r23g were significantly up-regulated by poly(l:C) in the spleen or/and kidney, which implies that the expanded TIr11 family genes may play roles in protecting the fish from the invasion of gram-negative bacteria and double-stranded RNA viruses. The results from the present study suggested that the expansion of TIr11 family genes in $B$. pectinirostris may recognize ligands from various pathogens found in the intertidal zone.

Keywords: Boleophthalmus pectinirostris, TLR21, TLR22, TLR23, innate immunity, positive selection, LPS, poly(I:C) 


\section{INTRODUCTION}

The Toll-like receptor (TLR) gene family is a class of pathogen recognition receptors (PRRs) that play crucial roles in the innate immune system by recognizing pathogen-associated molecular patterns (PAMPs) derived from various microbes $(1,2)$. TLRs interact with PAMPs from pathogens via their clusters of extracellular LRRs (leucine-rich repeats), resulting in conformational changes of TLRs. This further activates cytoplasmic Toll-Interleukin-1 receptor (TIR) domain to recruit cytosolic adaptor proteins, such as myeloid differentiation factor 88 (MyD88), and finally induces the production of multiple cytokines (3). Since the discovery of Toll in fruit fly (Drosophila melanogaster) in $1985(4,5)$, at least 28 TLRs have been identified in vertebrates and can be divided into six major families: TLR1 (TLRs 1, 2, 6, 10, 14, 15, 16, 18, 24, 25, 27 and 28), TLR3 (TLR3), TLR4 (TLR4), TLR5 (TLR5), TLR7 (TLRs 7, 8, 9), and TLR11 (TLRs 11, 12, 13, 19, 20, 21, 22, 23, and 26) (6). Bony fish are thought to have an ancient immune system and there is great scientific interest in comparing their innate and adaptive defense mechanism with mammals (7). So far, at least 21 Tlrs have been identified in fishes (8). TLR4 gene has been lost from the genomes of most fishes (9). TLR6 and TLR10 are absent in fishes (10). TLR5s, TLR14, TLR18 to TLR28 are considered to be termed as "teleost-specific TLRs" $(8,11)$.

Key features of the fish TLRs and the factors involved in their signaling cascade have high structural similarity to the mammalian TLR system. However, fish TLRs also exhibit very distinct features and large diversity which is likely derived from their diverse evolutionary history and the distinct environments that they occupy (7). In particular, some TLR genes in teleosts are known to be shaped by positive (diversifying or adaptive) selection, which enables them to cope with a large number of rapidly evolving pathogens (12-18).

The mudskipper Boleophthalmus pectinirostris (Linnaeus 1758 ) is a burrow-dwelling fish, widely distributing throughout the intertidal regions of China, Korea and Japan (19). B. pectinirostris is usually found on the soft mudflats of estuaries and coastal waters when they are exposed at ebb tide. Their behaviors, physiological and morphological features have been specialized and adapted for an amphibious lifestyle (20-25). Pathogenic bacteria adhere to and colonize mucosal surfaces of the susceptible host (26), or invade the body mainly through the skin, gill, or gut (27). The peculiar environment of the mudflats, which changes between flood and ebb tides, suggests that $B$.

Abbreviations: $\mathrm{Bp}$, Boleophthalmus pectinirostris; $\mathrm{CD}$, cluster of differentiation; CDS, complete codinig sequence; CpG-ODNs, CpG-oligodeoxynucletides; CT, Cterminus; MD-2, myeloid differentiation factor 2; eefl $\alpha$, eukaryotic translation elongation factor $1 \alpha$; hpi, hours post injection; LPS, lipopolysaccharide; LRRs, leucine-rich repeats; Map3k, mitogen-activated protein kinase kinase

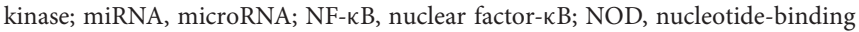
oligomerization domain; NT, N-terminus; PAMPs, pathogen-associated molecular patterns; poly(I:C), polyinosinic-polycytidilic acid; ppme1, protein phosphatase methylesterase-1; RIPK2, receptor-interacting serine-threonine kinase 2; SEM, standard error of the mean; Sh3kbp1, SH3-domain kinase binding protein 1; SRB, scavenger receptor class B; TIR, Toll-Interleukin-1 receptor; TLR, Tolllike receptor; TRIF, TIR-domain-containing adapter-inducing interferon- $\beta$; UTR, untranslated region. pectinirostris may have evolved specific immunity genes to adapt to their habitat. Interestingly, genomic study of $B$. pectinirostris showed that the fish species possesses the largest number (11 copies) of TLR13 in vertebrates sequenced so far (28). However, in the present study, based on the sequences of $11 \operatorname{Tlr} 13$ of $B$. pectinirostris, we further cloned one more Tlr13 gene from $B$. pectinirostris. However, the phylogenetic analysis indicated that the 12 Tlr13 from B. pectinirostris should be classified as Tlr21, Tlr22, and Tlr23, which belong to TLR11 family. Similar TLR11 family expansion was also reported in Atlantic cod (Gadus morhua), and 12 tlr22 paralogues of Atlantic cod responded differently to pathogenic challenge, which indicated that they are undergoing neofunctionalization via positive selection and can recognize bacterial pathogen-associated molecular patterns (13).

The aim of this study was to investigate if expansion of Tlr11 family in $B$. pectinirostris have been retained through adaptive evolution in order to provide special immune defense against pathogens from Vibrio, Klebsiella, Salmonella, etc. in intertidal mudflat $(29,30)$. After obtaining the full-length cDNA sequence of 12 Trr11 family genes in B. pectinirostris, we conducted synteny analysis and chromosome localization. In order to assess the adaptive evolution of Tlr11 family genes of $B$. pectinirostris, positive selection analysis was performed. We further examined the tissue distribution and the expression profiles of these genes in response to lipopolysaccharide (LPS) and polyinosinicpolycytidilic acid [poly(I:C)] challenges.

\section{MATERIALS AND METHODS}

\section{Experimental Fish and Sampling}

Adult mudskipper $B$. pectinirostris (body length 105$145 \mathrm{~mm}$, body weight $20-45 \mathrm{~g}$ ) used in this study were purchased from a seafood market in Xiamen, Fujian province, China. The fish were maintained in plastic tanks with $1.5 \mathrm{~cm}$ deep seawater at water temperature of $28-28.5^{\circ} \mathrm{C}$, and salinity of 15 ppt. Before sampling, the fish were anesthetized with $0.01 \%$ MS222 (Sigma-Aldrich, St. Louis, MO, US). All experiment protocols were approved by the Institute of Animal Care and Use Committee of Xiamen University.

\section{cDNA Cloning, Gene Structures of tIr21, t/r22, and t/r23 Paralogues}

The fasta format of whole genome shotgun sequences of $B$. pectinirostris was downloaded from NCBI, and a local blast database was created with BioEdit (31). The partial fragments of previous published 11 tr 13 genes of $B$. pectinirostris (28) were obtained from BGI (The Beijing Genomics Institute, Shenzhen), these sequences were used to search for the reference sequences from the database by BioEdit software. We found a new TLR11 family gene from local blast database based on the conserved cDNA sequence of TIR domain of B. pectinirostris. The start and stop codons of these genes were predicted by BLASTP searches (NCBI). Finally, we got sequences of 12 Tlr11 family genes in $B$. pectinirostris. Specific primers were designed to amplify the open reading frames of these genes (Supplementary Table 1). A cDNA library from kidney tissue was synthesized using the ReverAid First Strand cDNA Synthesis Kit (Thermo Scientific, USA) 
following the manufacturer's instructions and used to amplify these $12 \mathrm{tl}$ transcripts. Thirty-five cycles of standard PCR were performed on a Bio-Rad T100 Thermal Cycler, the annealing temperature was $58^{\circ} \mathrm{C}$ and the elongation time depended on the length of fragments amplified. No more than twenty cycles of nested PCR amplification with an annealing temperature of $58^{\circ} \mathrm{C}$ were performed when necessary.

The full-length end cDNA sequences of these $12 \mathrm{tl}$ genes were obtained using a SMART RACE cDNA Amplification kit (BD, Clontech) following the manufacturer's instructions, and the combined PCR sequences were used to deduce the full-length cDNA sequences of the $12 \mathrm{tl}$ genes. Briefly, total RNA extracted from the fresh kidney was used to synthesize the RACE Ready first-strand cDNA. The $3^{\prime} \mathrm{RACE}$ cDNA was synthesized using $3^{\prime}$-CDS Primer A, and the $5^{\prime}$ RACE cDNA was synthesized using $5^{\prime}$-CDS Primer $A$ and SMARTer IIA oligo. RACE primers for these 12 genes were designed based on the sequence information of the fragments obtained above (Supplementary Table 2). The PCR products were cloned into pMD19-T simple vector (TaKaRa Dalian, China) and sequenced by Invitrogen Ltd. (Guangzhou, China). Intron-exon boundaries of these 12 tlrs were identified using corresponding genome sequences and gene structure display server (http://gsds.cbi.pku. edu.cn/). Furthermore, we searched for the highly conserved tandem repeat sequences in the full-length cDNA sequences of these 12 tlrs from $B$. pectinirostris using the online software “Tandem Repeats Finder" (32).

\section{Synteny Analysis and Chromosome Location of t/r21, t/r22, and t/r23 Paralogues}

Synteny analysis was performed manually based on the genome assemblies of large yellow croaker (Larimichthys crocea) (genome assembly accession no. GCF_000972845.1), green-spotted pufferfish (Tetraodon nigroviridis) (GCA_000180735.1), tiger pufferfish (Takifugu rubripes) (GCF_000180615.1), yellowtail kingfish (Seriola lalandei dorsalis) (GCA_002814215.1), amberjack (Seriola dumerili) (GCF_002260705.1), Asian seabass (Lates calcarifer) (GCF_001640805.1).

A high-quality chromosome map comprising $916.23 \mathrm{Mb}$ (93.2\%) of B. pectinirostris entire sequence was constructed as part of our program, and will be published separately (data not shown). The full-length cDNA sequences of these $12 \mathrm{tl}$ genes were used to determine their locations in the 23 pseudochromosomes of $B$. pectinirostris by BLAST searches (https:// blast.ncbi.nlm.nih.gov/Blast.cgi).

\section{Phylogenetic Analyses}

The deduced amino acid sequences of the expanded Tlr11 family genes were obtained using the ExPASy Translate Tool (http://www.expasy.ch/tools/dna.html). Protein domains, signal peptide, and transmembrane regions were predicted using SMART (http://smart.embl-heidelberg.de/), SignalP 4.1 (http:// www.cbs.dtu.dk/services/SignalP/) and the TMHMM Server v. 2.0 (http://www.cbs.dtu.dk/services/TMHMM/), respectively. A homology search was performed using the BLAST tool at
NCBI (http://www.ncbi.nlm.nih.gov/BLAST/). The phylogenetic reconstruction was performed using MEGA software 7 (33) by the Neighbor-joining method, and a bootstrap consensus tree was inferred from 1,000 replicates. We also constructed a maximum likelihood phylogenetic tree in MEGA software 7 (33) using the Tamura 3-parameter model and $\gamma$ distributed rates with invariant sites $(\mathrm{G}+\mathrm{I})$ and $5 \gamma$ categories, and a bootstrap consensus tree was inferred from 1,000 replicates. GenBank accession numbers of $t$ r genes for alignment of amino acids and phylogenetic tree construction are as follows: Anser cygnoides TLR21 (AMB20882); Gallus gallus TLR21 (NP_001025729); Epinephelus coioides TLR21 (AEK49148); Takifugu rubripes TLR21 (AAW69371); Oreochromis niloticus TLR21 (AHK13949.1); Gadus morhua TLR21 (AFK76484.1); Salmo salar TLR21 (CDH93614.1); Danio rerio TLR21 (CAQ13807); Anolis carolinensis TLR21 (XP_008123135.2); Xenopus tropicalis TLR21 (XP_002936443.2); Epinephelus coioides TLR22 (AGA84053.1); Scophthalmus maximus TLR22 (AIC75881.1); Takifugu rubripes TLR22 (AAW69372.1); Larimichthys crocea TLR22 (XP_010741403); Tetraodon nigroviridis TLR22 (CAG05452.1); Gadus morhua TLR22b (AFK76486.1); Gadus morhua TLR22d (AFK76488.1); Gadus morhua TLR22g (AFK76491.1); Gadus morhua TLR22i (AFK76493.1); Miichthys miiuy TLR23 (ALJ55575.1); Takifugu rubripes TLR23 (AAW70378.1); Gadus morhua TLR23a (AFK76497.1); Gadus morhua TLR23b (AFK76498.1); Labrus bergylta TLR23 (XP_020513361.1); Tetraodon nigroviridis TLR23 (CAF93842.1); Seriola dumerili TLR23a (XP_022616855.1); Seriola dumerili TLR23b (XP_022603128.1); Seriola dumerili TLR23c (XP_022603127.1); Lates calcarifer TLR23a (XP_018537426.1); Lates calcarifer TLR23b (XP_018546010.1); Lates calcarifer TLR23c (XP_018517760.1); Seriola lalandi dorsalis TLR23a (XP_023286622.1); Seriola lalandi dorsalis TLR23b (XP_023252716.1); Seriola lalandi dorsalis TLR23c (XP_023252714.1).

\section{Analyses of Positive Selection}

The complete coding sequences (CDS) of the 12 Tlr11 family genes from $B$. pectinirostris (Table 1) were first aligned with MUSCLE (www.ebi.ac.uk/Tools/msa/muscle) and a codon alignment was obtained using RevTrans 2.0b (www.cbs.dtu.dk/ services/RevTrans-2.0/web) followed by Codon Align (www. hiv.lanl.gov). The $\mathrm{N}$ - and $\mathrm{C}$ - terminal portions (60 and 12 codons, respectively) of the codon aligned TLR11 sequences were too variable and hence not included in the following analysis. Gaps present in more than one sequence were also manually removed. The refined codon alignment used in the selection tests comprised $93 \%$ of the total CDS and did not have any stop codons. This alignment was used to construct a maximum likelihood phylogenetic tree in MEGA7 (33) using the Tamura 3 -parameter model and $\gamma$ distributed rates with invariant sites $(\mathrm{G}+\mathrm{I})$ and $5 \gamma$ categories. A bootstrap consensus tree was inferred from 1,000 replicates.

The average number of synonymous and non-synonymous (amino acid-changing) substitutions, insertions and deletions in the codon alignments were calculated using SNAP (www. hcv.lanl.gov). This algorithm performs pairwise comparisons between all sequences in the alignment using the method 
TABLE 1 | The characterization of t/r21, t/r22, and t/r23 paralogues in B. pectinirostris.

\begin{tabular}{|c|c|c|c|c|c|c|c|c|}
\hline Gene name & Full cDNA (bp) & ORF (bp) & 5'UTR (bp) & 3'UTR (bp) & No. of exons & Number of amino acids & Scaffold & Genbank accession no. \\
\hline $\operatorname{tr} 21$ & 3520 & 2898 & 255 & 367 & 1 & 965 & scaffold9 & $\mathrm{MH} 744540$ \\
\hline t/r22a & 3820 & 2853 & 72 & 895 & 4 & 950 & scaffold291 & $\mathrm{MH} 744541$ \\
\hline $\operatorname{tr} 22 b$ & 3559 & 2697 & 165 & 697 & 4 & 898 & scaffold890 & MH744542 \\
\hline tIr22c & 3219 & 2877 & 74 & 268 & 4 & 958 & scaffold183 & $\mathrm{MH} 744543$ \\
\hline $\operatorname{tr} 22 d$ & 4637 & 2856 & 1524 & 257 & 3 & 951 & scaffold103 & $\mathrm{MH} 744544$ \\
\hline t/r23a & 3694 & 2814 & 194 & 686 & 5 & 937 & scaffold936 & $\mathrm{MH} 744545$ \\
\hline trr23b & 2878 & 2748 & 45 & 85 & 4 & 915 & scaffold1155 & $\mathrm{MH} 744546$ \\
\hline $\operatorname{tir} 23 c$ & 4825 & 2883 & 37 & 1905 & 5 & 960 & scaffold294 & MH744547 \\
\hline $\operatorname{tir} 23 d$ & 4904 & 2820 & 436 & 1648 & 4 & 939 & scaffold1045 & MH744548 \\
\hline t/r23e & 4410 & 2766 & 1410 & 234 & 3 & 921 & scaffold219 & $\mathrm{MH} 744549$ \\
\hline tIr23f & 3451 & 2805 & 37 & 609 & 5 & 934 & scaffold294 & $\mathrm{MH} 744550$ \\
\hline t/r23g & 3574 & 2835 & 9 & 730 & 5 & 944 & scaffold50 & MH744551 \\
\hline
\end{tabular}

developed by Nei and Gojobori (34). In protein coding genes, the ratio $(\omega)$ between non-synonymous $(\mathrm{dN})$ and synonymous (dS) substitution rates is related to evolutionary constraints at the protein level (35). A value of $\omega>1$ indicates positive Darwinian selection, whereas $\omega<1$ suggests negative or purifying selection. A codon based Z-test of selection was performed to test the hypothesis of positive selection in MEGA7 (33) using the modified Nei-Gojobori method with Jukes-Cantor correction (36). The hypothesis of positive selection was further tested using the likelihood methods implemented in the CODEML program of PAML v4.9 (37) and the Datamonkey adaptive evolution server (38), as detailed elsewhere (39). In PAML, the data set was fitted to 6 models of codon substitution: M0 (one ratio), M1 (two site classes), M2 (positive selection with three site classes, M3 (discrete), M7 ( $\beta$ ) and M8 (continuous). Bayesian posterior probabilities were calculated for positively selected sites using naïve empirical Bayes in the case of model M3 or Bayes empirical Bayes for models M2 and M8. Likelihood ratio tests were used to compare the corresponding models with and without selection (i.e., M2 vs. M1, M3 vs. M0, and M8 vs. M7). FEL, SLAC and REL analyses were performed in Datamonkey to calculate $\mathrm{dN}-\mathrm{dS}$ values for each codon, along with the corresponding probability values. Overall differences in diversifying selection between paralogous genes were determined with GA-branch method implemented in Datamonkey.

The three-dimensional structure of $B$. pectinirostris Tlr23a was predicted by SWISS-MODEL (40). In brief, structural template searches against the SWISS-MODEL template library were performed with BLAST and HHBlit. The highest quality template was then selected for model building based on the target-template alignment using ProMod3. The global and per-residue model quality has been assessed using the QMEAN scoring function (41). For improved performance, weights of the individual QMEAN terms have been trained specifically for SWISS-MODEL. The local quality plot was shown in Supplementary Figure 1. Positively selected codons were identified in the threedimensional protein using the web-based viewer $\mathrm{iCn} 3 \mathrm{D}$ at NCBI (www.ncbi.nlm.nih.gov/Structure/icn3d/full.html).

\section{Expression of t/r21, t/r22, and t/r23 Paralogues in Different Tissues}

To further explore the potential functions of $t / r 21, t \operatorname{lr} 22$, and tlr23 paralogues in B. pectinirostris, the basal expression levels of these 12 Tlr11 family genes in different tissues were quantified by real-time qPCR. Tissues including brain, heart, spleen, gills, liver, intestine, testis, seminal vesicle, ovary, skin, eye, kidney, blood cells were collected separately from seven $B$. pectinirostris. All the samples were snap-frozen in liquid nitrogen and stored at $-80^{\circ} \mathrm{C}$ until analyses. Total RNA extraction, cDNA synthesis and real-time qPCR were performed as described in section Real-Time qPCR.

\section{Expression of t/r21, t/r22, and t/r23 Paralogues in Response to LPS and Poly(l:C) Challenges}

LPS is the main component of the cell surface of Gram-negative bacteria and poly(I:C) is used here as a model of double-stranded RNA virus infection. To investigate the potential functions of the 12 Tlr11 family genes in B. pectinirostris, the expression levels of these genes in the spleen and kidney were analyzed following intraperitoneal injections of LPS and poly(I:C). Male B. pectinirostris with similar size (body length $119-132 \mathrm{~mm}$, body weight 29.5-33.6g) were transported live in plastic tanks and acclimated to laboratory conditions (seawater at salinity $15 \mathrm{ppt}$ and temperature $28-28.5^{\circ} \mathrm{C}$ ) for 1 day. For the LPS challenge experiment, fish were randomly divided into two groups and each fish was intraperitoneally injected with LPS (Sigma, E. coli 0127:B8) dissolved in sterile $100 \mu \mathrm{L}$ PBS at the dose of $0.1 \mathrm{mg}$ in the treated group or with $100 \mu \mathrm{L}$ sterile PBS in the control. At 3, 6, 12, $24 \mathrm{~h}$ post injection (hpi), the spleen and kidney of five or six individuals from each group at each time point were surgically sampled, frozen immediately in liquid nitrogen and stored at $-80^{\circ} \mathrm{C}$ until analyses. For the poly(I:C) challenge experiment, the fish were prepared as described above. Each fish was intraperitoneally injected with poly(I:C) (Sigma, P0913) dissolved in sterile $100 \mu \mathrm{L}$ PBS at the dose of $0.1 \mathrm{mg}$ in the treated group or with $100 \mu \mathrm{L}$ sterile PBS in the control group. The spleen 
and kidney of five or six individuals from each group at 3, 6, 12 , and 24 hpi were surgically collected. Total RNA extraction and CDNA synthesis of these organs were conducted as described in section Real-Time qPCR. Real-Time qPCR was performed as described in section Real-Time qPCR.

\section{Real-Time qPCR}

Total RNA was extracted from tissues using the RNAiso Plus (TaKaRa Dalian, China) and treated with RNase-free DNase I (Fermentas, USA) to eliminate contaminated genomic DNA. $1.5 \mu \mathrm{g}$ total RNA was used for the synthesis of the first strand cDNAs using the RevertAid first stand cDNA synthesis kit (Thermo Scientific, USA). The gene specific primers used for real-time $\mathrm{qPCR}$ analysis and amplicon lengths are listed in Table 2. Amplification was conducted on a qTOWER 2.2 Real-Time PCR (Analytik Jena AG, Jena, Germany) using the PowerUp SYBR Green Real-time PCR Master Mix kit (Thermo Scientific, USA). Each $20 \mu \mathrm{L}$ reaction contained $10 \mu \mathrm{L}$ of PowerUp SYBR Green Real-time PCR Master Mix, $2 \mu \mathrm{L}$ of cDNA template, $1 \mu \mathrm{L}$ of each primer $(10 \mu \mathrm{M})$, and $6 \mu \mathrm{L}$ of water. Sterilized water was substituted for the cDNA in negative control samples. The amplification program was performed as follows: predenaturation at $95^{\circ} \mathrm{C}$ for $2 \mathrm{~min}$ followed by 40 cycles at $95^{\circ} \mathrm{C}$ for $15 \mathrm{~s}, 60^{\circ} \mathrm{C}$ for $30 \mathrm{~s}$, and $72^{\circ} \mathrm{C}$ for $30 \mathrm{~s}$. Each sample was analyzed in duplicate.

\section{Statistical Analysis}

Statistical analysis was performed using Graphpad software and the relative abundance of mRNA for target genes was calculated using $2^{-\Delta \Delta C t}$ method (42) with the eukaryotic translation elongation factor $1 \alpha$ (eef1 $\alpha$, Genbank accession No. XM_020932525.1) gene as the reference. Data were presented as mean \pm standard error of the mean (SEM) and the Student's $t$-test was used to assess statistical differences of expression levels between groups. For multiple group comparison, oneway ANOVA followed by Tukey's test was used for statistical analysis. Differences were considered to be statistical significance when $p<0.05$.

\section{RESULTS}

\section{cDNA Sequences of t/r21, t/r22, and t/r23 Paralogues}

The characterization of $t \operatorname{lr} 21, t \operatorname{tr} 22$, and $t \operatorname{lr} 23$ paralogues is summarized in Table $\mathbf{1}$ and their structures are showed in Figure 1, based on the genome assembly of $B$. pectinirostris. Although the lengths of these $12 \mathrm{tr}$ cDNAs varied from 2,878 to $4,904 \mathrm{bp}$, the deduced protein sizes were conserved, ranging from 898 to 965 amino acids. With 3,520 bp, the tlr21 full cDNA was encoded by a single exon, including 255 bp $5^{\prime}$-UTR, 367 bp $3^{\prime}$ UTR, and the 2,898 bp complete coding region corresponding to a 965 aa protein. Among tlr 22 and t tr 23 orthologs, only tlr $22 d$ and tlr23e comprised 3 exons and had longer $5^{\prime}$-UTRs, while tlr23c and tlr23d contained longer $3^{\prime}$-UTRs.

Several highly conserved tandem repeats were found mainly within $5^{\prime}$-UTR or $3^{\prime}$-UTR of tlr22b, tlr $23 a, t \operatorname{tr} 23 d$ (Supplementary Table 3) and tlr22d (Supplementary Figure 2).
TABLE 2 | The primers for Real-time qPCR in this study.

\begin{tabular}{|c|c|c|}
\hline Primer & Sequence $\left(5^{\prime}-3^{\prime}\right)$ & Amplicon length (bp) \\
\hline t/r21 rtF & AACTCTGTCTACATCACAGAGAC & 272 \\
\hline trr21 rtR & CAGATAGGTCTTCTTAAGCATGAC & \\
\hline t/r22a rtF & CTGGAGAACGATCAAGGCTGGAAG & 146 \\
\hline t/r22a rtR & CACTCGCTCTGTAGATATCGTCTG & \\
\hline t/r22b rtF & TTCAGCAGATTTCACCTGAGTTAC & 241 \\
\hline t/r22b rtR & CTTCAGCGATGTTCTCCACGATG & \\
\hline t/r22c rtF & TATAGAGAACTAGTGCCACATCTG & 214 \\
\hline t/r22c rtR & GCTCATCGAACAAACGGAAACTG & \\
\hline tlr22d rtF & GCGTAGAGGATCAGTACGATG & 178 \\
\hline t/r22d rtR & CTTCCATAAATGGCATCAGCAATG & \\
\hline t/r23a rtF & GCTGGAGGCTTTGTCTGCACCAC & 186 \\
\hline t/r23a rtR & CGTCTITGTGCTCATCGAACAGAC & \\
\hline tr23b rtF & CGTTCGTTTCCTACAACGTTCACG & 263 \\
\hline t/r23b rtR & CACGTCCTTCTGCTCATCGAACAG & \\
\hline t/r23c rtF & 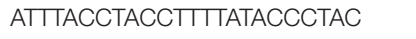 & 321 \\
\hline t/r23c rtR & GCTCAAGTACAAATAGAGTCAATC & \\
\hline t/r23d rtF & GTTTCTAAGGACAAAGCTCATGAC & 247 \\
\hline t/r23d rtR & CACAGACTTATITTGGAGCATCTG & \\
\hline tir23e rtF & TGTGTCCTACAACTGTCACGATG & 167 \\
\hline t/r23e rtR & ACAGAGTCTTCCTGCTTCTGTAG & \\
\hline t/r23f rtF & AACAACAAAATTGATCATATTTCC & 248 \\
\hline t/r23f rtR & CGAAATTTGGTCAAAGTATCTGAG & \\
\hline t/r23g rtF & CTACAACGTTCATGATGAGAACTG & 251 \\
\hline t/r23g rtR & САTССТTСТGСТСАTCAAACAGAC & \\
\hline eef1 $\alpha$ rtF & TGGAACCTCTCAGGCTGACT & 275 \\
\hline eef1 $\alpha \mathrm{rtR}$ & ATCCAGAGATGGGCACAAAG & \\
\hline
\end{tabular}

$F$, forward; $R$, reverse.

The longest tandem repeats spanning 563 bp were identified in the $5^{\prime}$-UTR of $t$ tr $22 d$, and the shortest tandem repeats spanning 96 bp were found in the $5^{\prime}$-UTR of trr22b. The copy number of $11 \mathrm{bp}$ tandem repeat within the $3^{\prime}$-UTR of tlr22b was up to 50. The tandem repeats of tlr23a started at the $5^{\prime}$ end of the cDNA. The tandem repeats of tlr $23 d$ were completely distributed within $3^{\prime}$-UTR.

\section{Synteny Analysis and Chromosome Location of t/r21, t/r22, and t/r23 Paralogues}

Both $t$ tr23c and tlr23f were present in the same scaffold (scaffold294), while the other paralogues were mapped to different scaffolds (Table 1; Figure 2A). Partial synteny analysis based on the current mudskipper genome build revealed conservation between tlr22a in B. pectinirostris and tlr22 coding genes in large yellow croaker, green-spotted pufferfish and tiger pufferfish, within the genomic region containing sh $3 k b p 1$, map3k15, and cnksr2 (Figure 2B). Tlr23a in B. pectinirostris and tlr23as in yellowtail kingfish, amberjack and Asian seabass were adjacent with the same gene "ppme1-like" (Figure 2B).

All these $12 \mathrm{tlr}$ genes, except tlr23b, mapped on seven chromosomes of B. pectinirostris (Figure 3, 


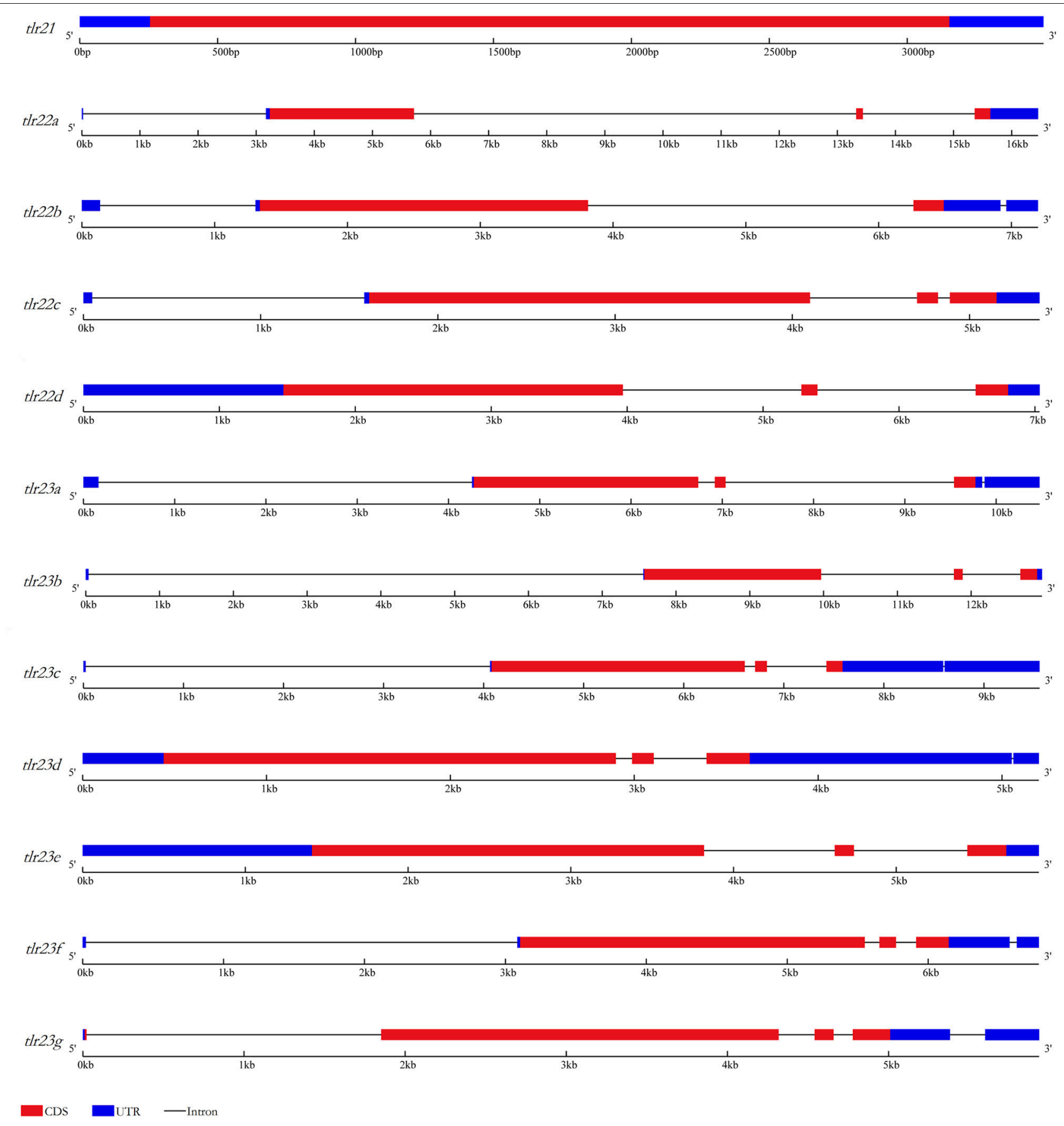

FIGURE 1 | Gene structures of t/r21, trr22, t/r23 paralogues in B. pectinirostris. Graphical representation of trr21, trr22, and t/r23 gene structures. UTRs and CDS are represented in light blue and red, respectively. Introns are indicated by continuous lines.

Supplementary Table 4). Tlr21 was located at chr7, thr22a, $\operatorname{tl} 22 b, \operatorname{tl} 22 c$, and $\operatorname{tl} 22 d$ were located at chr12, chr8, chr7, and chr17, respectively. Among tlr23 genes, three of them (tlr23c, $t l r 23 d, t l r 23 f)$ were present in the same chromosomal region (chr11), while tlr23a and $t$ tr23g were found in chr6 and chr18, respectively. The location of $t r 23 b$ needs to be further explored.

\section{Phylogenetic Analysis and Protein Domain} Arrangements of $t / r 21, t / r 22$, and tIr23i Paralogues in B. pectinirostris

The phylogenetic tree was constructed using the Neighborjoining method on the basis of deduced amino acid sequences of TLR21, TLR22, and TLR23 of vertebrates (Figure 4). 


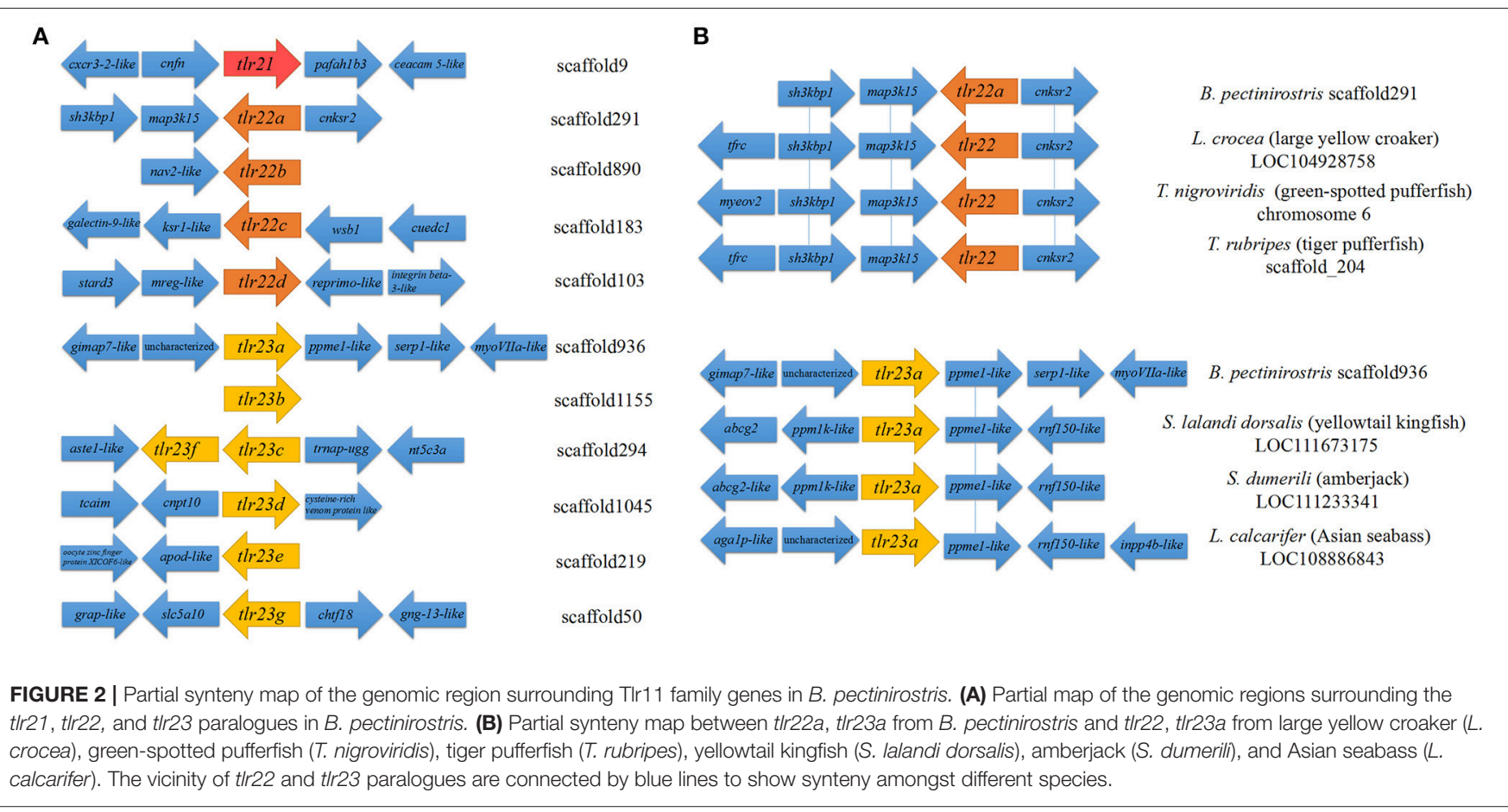

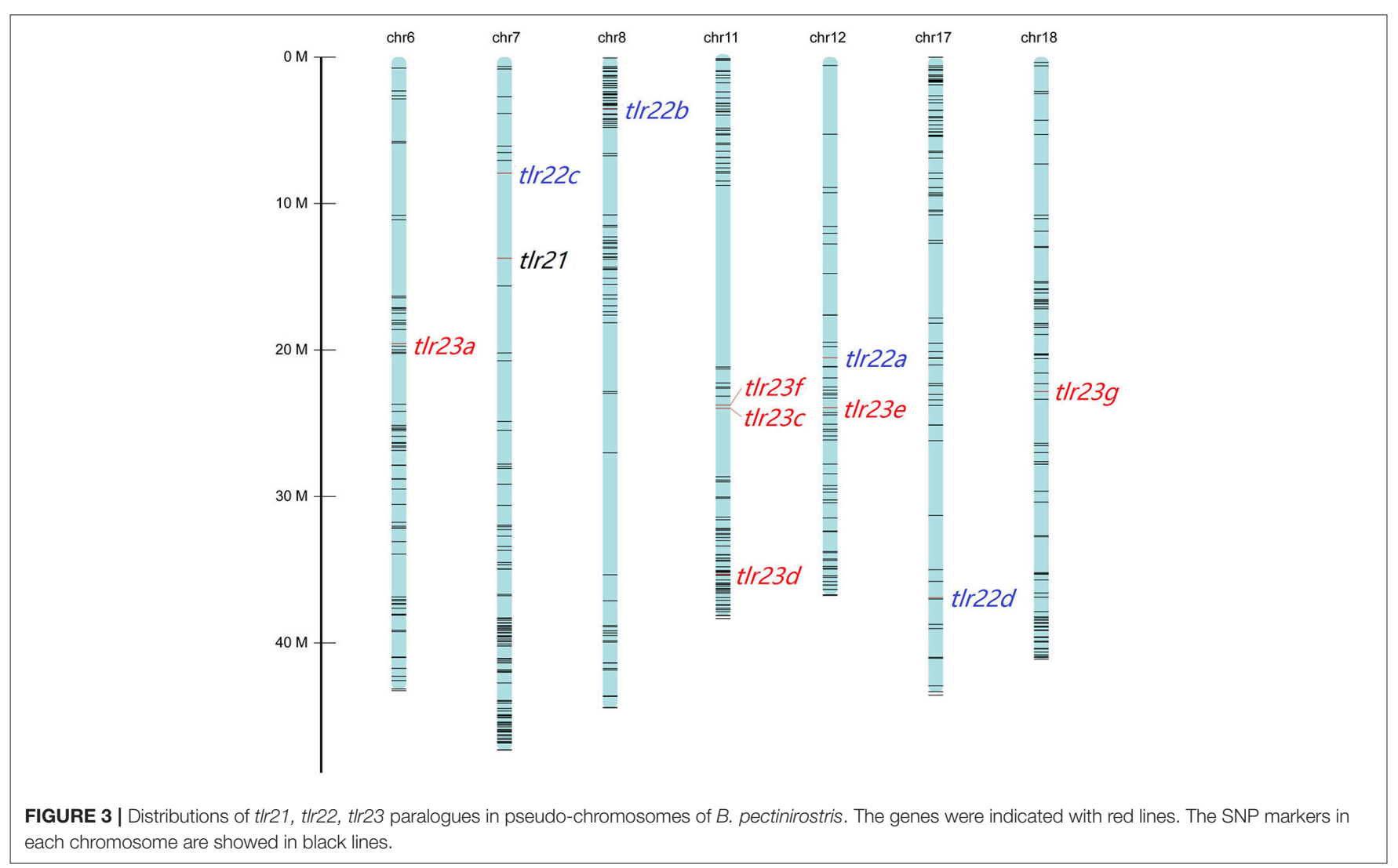

Phylogenetic analysis showed that TLR21, TLR22, and TLR23 constituted three major groups. In the tree, all TLR21 were grouped under a single clade, while TLR22 and TLR23 formed a separate cluster. The Tlr22 orthologs in B. pectinirostris were grouped together. All Tlr23 orthologs except Tlr23a clustered together in $B$. pectinirostris. Similar results were also 


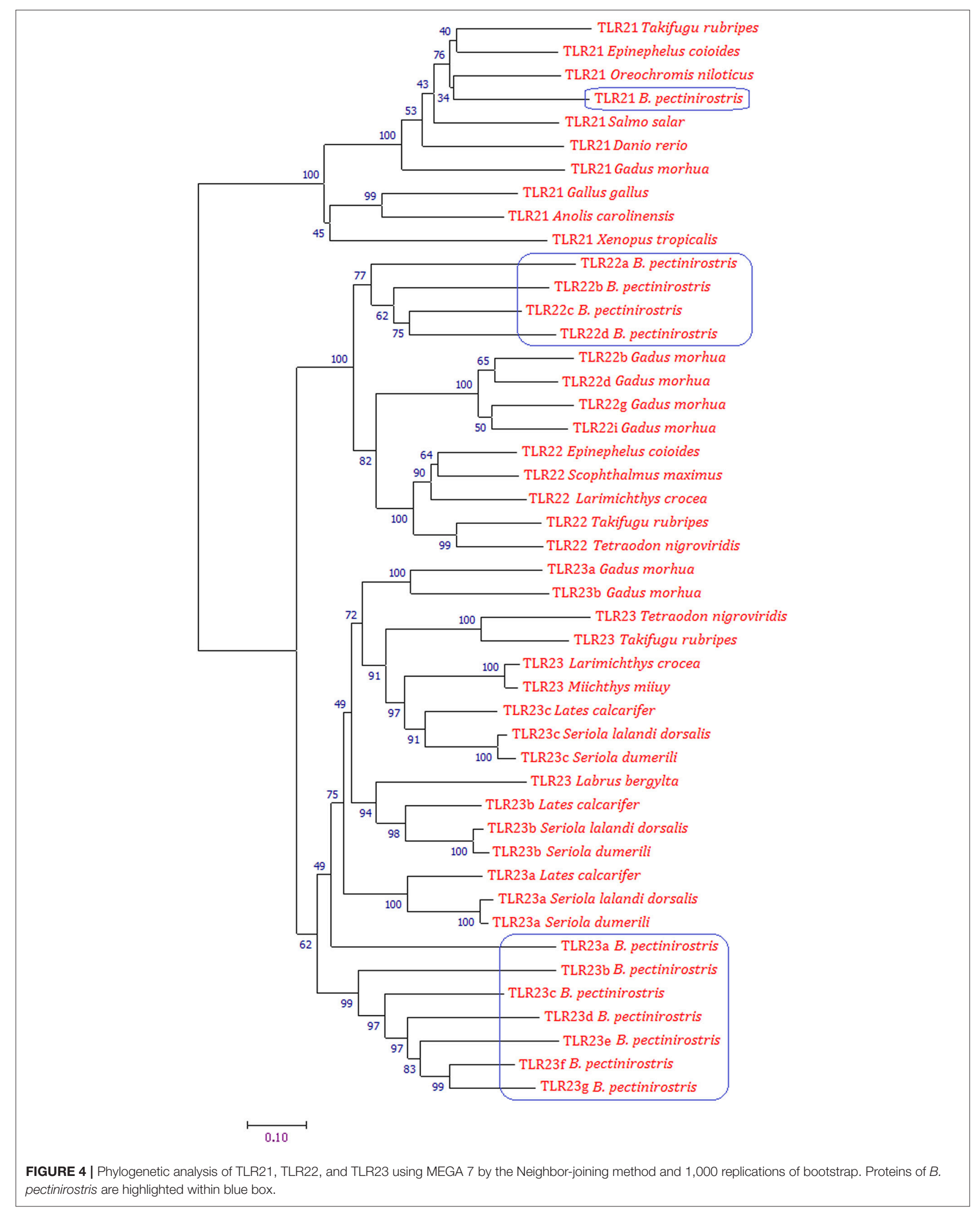



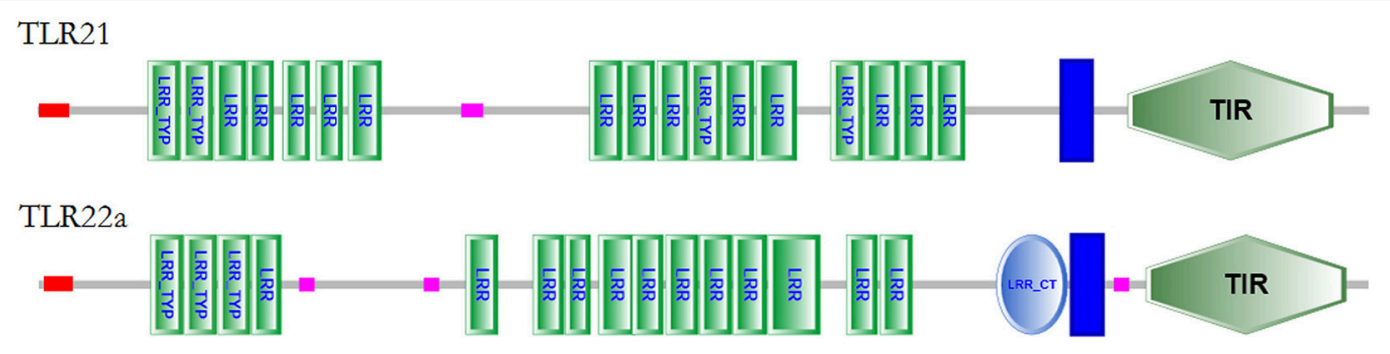

TLR22b

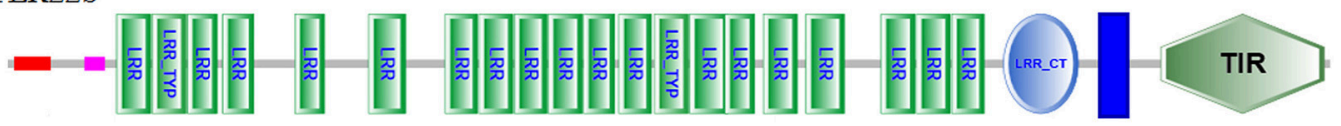

TLR22c
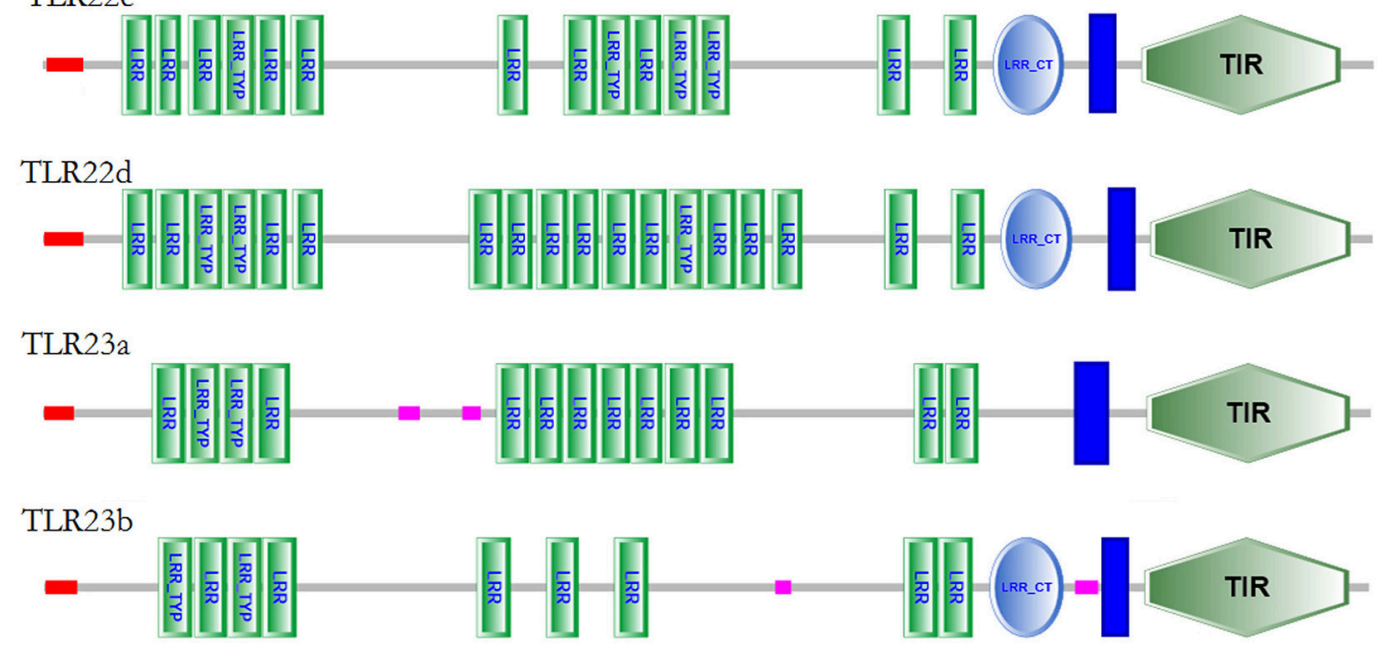

$\operatorname{TLR} 23 \mathrm{c}$
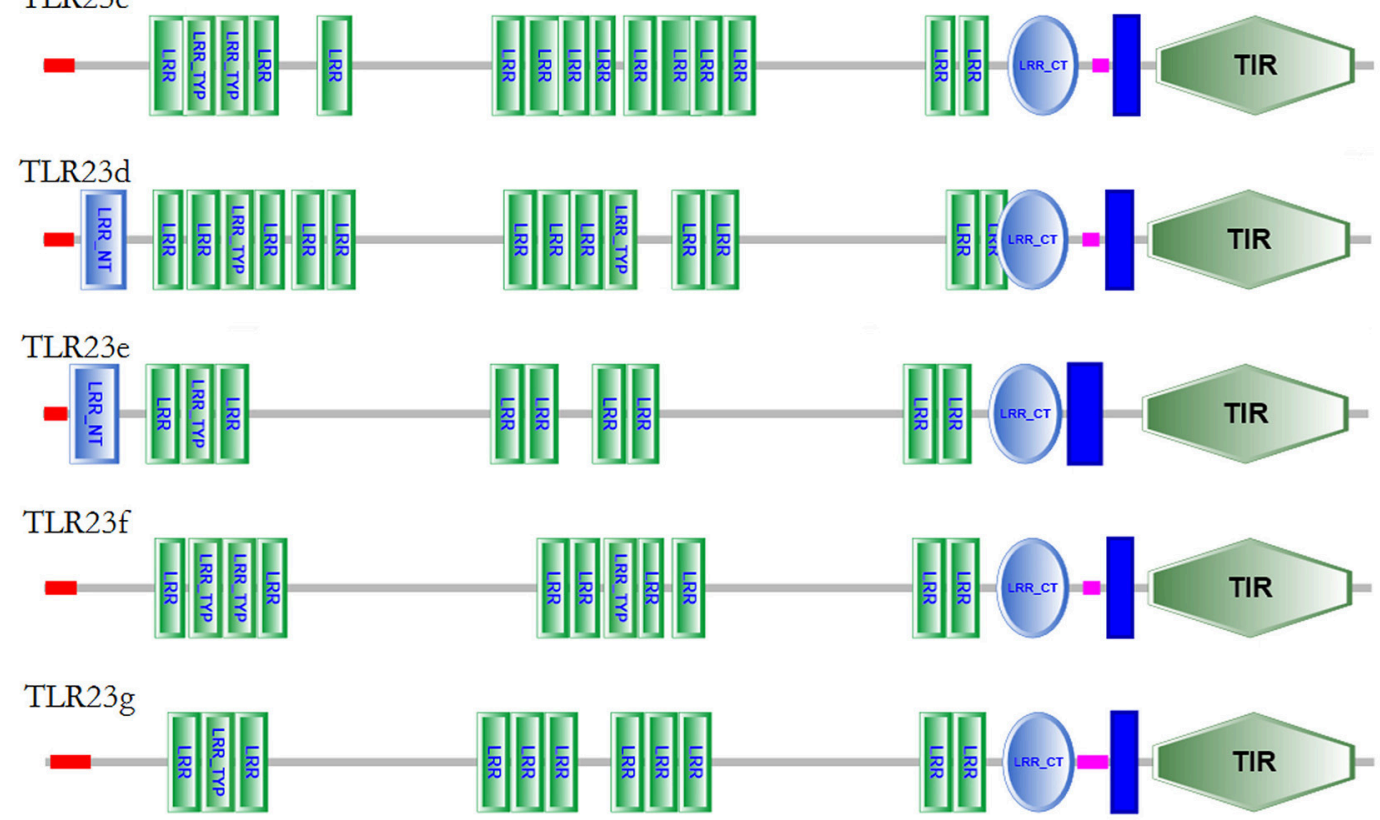

FIGURE 5 | Protein domain structures of TIr21, TIr22, and TIr23 proteins in B. pectinirostris. The domain organizations of Tlr21, TIr22, and Tlr23 in B. pectinirostris were predicted using SMART, SignaIP, and TMHMM analyses. LRR, leucine-rich repeat; LRR-TYP, leucine-rich repeat typical subfamily; TIR, Toll/L-1 receptor; NT, $\mathrm{N}$-(nitrogen) terminal; CT, C-(carboxyl) terminal; $\mathbf{\square}$, signal peptide; $\mathbf{\square}$, transmembrane region; $\mathbf{\square}$, low complexity region. 
found in the phylogenetic tree based on Maximum Likelihood method (Supplementary Figure 3).

The protein domain arrangements of $t l r 21, t l r 22$, and $t l r 23$ paralogues in B. pectinirostris are presented in Figure 5. All Tlrs amino acid sequences comprised of a signal peptide, several LRRs, a transmembrane domain and a TIR domain. These Tlrs contained various numbers of LRR domains, and the LRR number in each TLR ranged from 10 to 21: 17 (Tlr21), 16 (Tlr22a), 21 (Tlr22b), 15 (Tlr22c), 19 (Tlr22d), 13 (Tlr23a), 10 (Tlr23b), 16 (Tlr23c), 16 (Tlr23d), 11 (Tlr23e), 12 (Tlr23f), 12 (Tlr23g). Most of these Tlrs had C-terminus LRRs (LRRCT) with the exception of Tlr21 and Tlr23a. Only Trr23d and Tlr23e contained N-terminus LRRs (LRR-NT). Besides, Tlr22b contained a shorter TIR domain than other Tlrs.

\section{Molecular Evolution of the TIr11 Family in B. pectinirostris}

A sliding window analysis of the complete coding sequences of tlr21, tlr22, and $t \operatorname{tr} 23$ paralogues revealed that the cumulative number of non-synonymous mutations per codon $(\mathrm{dN})$ exceeded the number of synonymous substitutions (dS) and that their occurrence was not uniform throughout the tlr21, tlr22, and tlr23 coding sequences (Figure 6). The average dS of all pairwise comparisons was higher than $\mathrm{dN}$ but different between the LRR and TIR domains, with $\mathrm{dS} / \mathrm{dN}$ ratios of 4.044 and 6.933 , respectively. A pairwise codon-based $Z$-test did not reject the null hypothesis of strict-neutrality $(\mathrm{dN}=\mathrm{dS})$ in favor of positive selection (dN > dS) (Supplementary Table 5). Nevertheless, the $\mathrm{dN} / \mathrm{dS}$ ratios $(\omega)$ varied between tlr11 genes

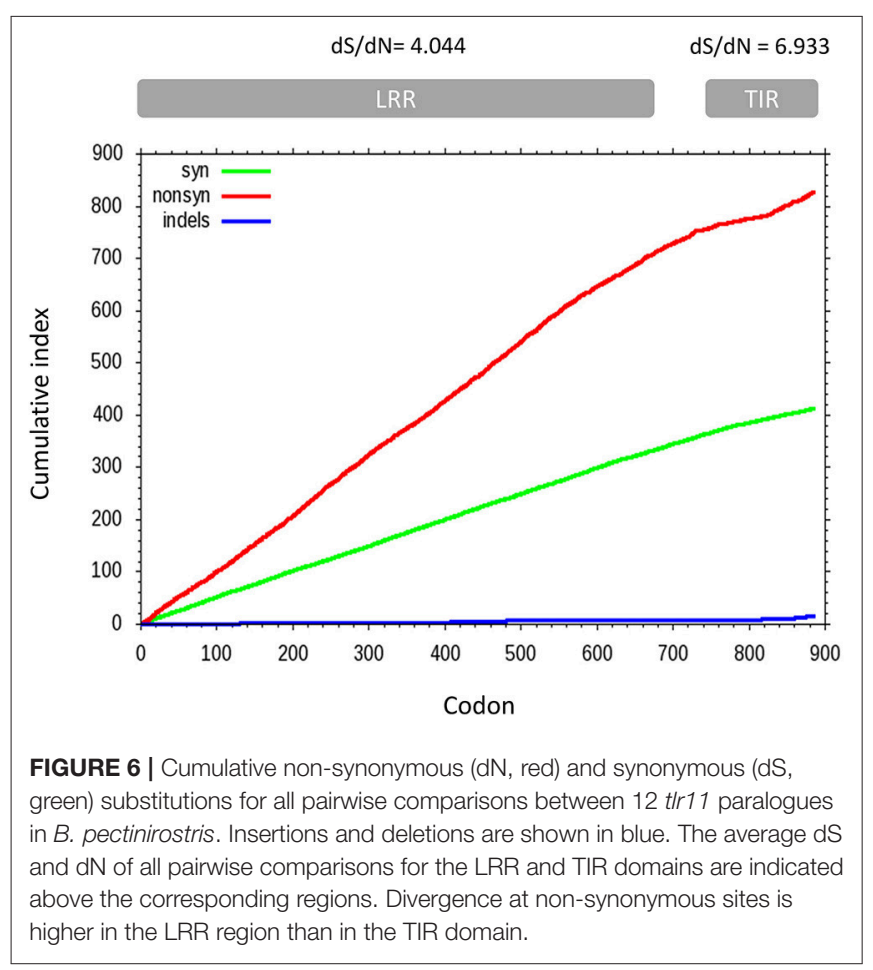

and were highest in $t \operatorname{lr} 23$ paralogues, particularly in $t l r 23 c-g$ (Supplementary Figure 4).

A more detailed site-specific analysis was performed using likelihood models to identify codons under diversifying selection. Likelihood ratio tests in PAML showed that models allowing for positive selection fitted the data better than those that did $\operatorname{not}(\mathrm{M} 3$ vs. $\mathrm{M} 0,2 \Delta \mathrm{LnL}=1614, p=0 ; \mathrm{M} 2$ vs. $\mathrm{M} 1,2 \Delta \mathrm{LnL}$ $=45, p=0$; M8 vs. M7, $2 \Delta \operatorname{LnL}=40.6, p=0$ ) (Table 3). Models, M2, M3, and M8 identified 4, 46 and 3 codons under positive selection (Bayesian posterior $p<0.05$ ) and $\omega$ values of $5.39,1.02$, and 2.8 , respectively. The best model of nucleotide substitution was 012032 with an Akaike information criterion of 63195. FEL and SLAC analyses found 18 and 3 codons under positive selection with $p<0.1$ and REL identified 6 positively selected sites with Bayes factor $>50$ (Table 3). In total, 13 codons were identified by more than one likelihood model as being under significant positive selection pressure. In particular, codon 68 (F, $\mathrm{H}, \mathrm{K}, \mathrm{N}, \mathrm{R}, \mathrm{S}, \mathrm{T}, \mathrm{V}$, or W) was flagged by all models, except REL (Supplementary Figure 5). Only two out of these 13 codons were present within the TIR domain, whereas 11 were found in the ectodomain. Most positively selected sites (8 out of 13) were found in LRR regions, especially in the coils on the convex surface of this horseshoe-shaped domain. Two sites under diversifying selection (405 and 597) were located in beta sheets within the concave surface (Figure 7, Supplementary Figure 5).

\section{Tissue Distribution of t/r21, t/r22, and t/r23 Paralogues in $B$. pectinirostris}

$T l r 21, t l r 22$, and $t \operatorname{lr} 23$ paralogues showed distinguishable tissue expression patterns (Figure 8). They were all expressed in immune-related organs, i.e., spleen and kidney. In addition, tlr21 was widely distributed and predominantly expressed in the brain, testis and eye (Figure 8A). Among tlr22 paralogues (Figures 8B-E), only tlr22c was detectable in all tissues examined (Figure 8D). Compared with tlr21 and tlr22, tlr23 paralogues were exclusively expressed in the spleen and kidney (Figures 8F-L). The expression of these $t l r$ genes in the seminal vesicle, ovary and skin was weak.

\section{Expression of the t/r21, t/r22, and t/r23 Paralogues in Response to LPS and Poly(I:C) Challenges}

The expression of $t / r 21, t t r 22$, and $t$ tr23 paralogues responsed differently to LPS (Figures 9, 10) and poly(I:C) (Figures 11, 12) challenges. The expression of tlr21 was significantly upregulated by LPS in the spleen and kidney at 12 hpi (Figures 9A, 10A). Among tlr22 paralogues, LPS administration significantly stimulated tlr22b in the spleen at $3 \mathrm{hpi}$ (Figure 9C), and down regulated trr22a in the spleen at 6 hpi (Figure 9B) and $\operatorname{ttr} 22 \mathrm{c}$ in the kidney at $6 \mathrm{hpi}$ (Figure 10D). However, the expression profiles of these genes didn't show time-dependent significant differences. In response to poly(I:C) administration, tlr22a and tlr22d in the spleen showed clear significantly timedependent increase pattern, and reached the significantly highest levels at $12 \mathrm{hpi}$ (Figures 11B,E). In the kidney, only tlr22a significantly increased at $6 \mathrm{hpi}$ after poly(I:C) stimulation, and 
TABLE 3 | Positively selected sites in mudskipper t/r21, t/r22, and t/r23 paralogues.

\begin{tabular}{|c|c|c|c|c|c|}
\hline Method & Model & Parameter estimates & Ln likelihood & Model comparison & Positively selected sites ${ }^{a}$ \\
\hline \multirow[t]{6}{*}{ CODEML $^{\mathrm{b}}$} & MO: neutral & $\omega=0.32$ & -30533.3 & & None \\
\hline & $\begin{array}{l}\text { M1: nearly } \\
\text { neutral }\end{array}$ & $\begin{array}{l}\omega_{0}=0.15, \omega_{1}=1.00 \\
p_{0}=0.50, p_{1}=0.50\end{array}$ & -29907.2 & & Not allowed \\
\hline & $\begin{array}{l}\text { M2: positive } \\
\text { selection }\end{array}$ & $\begin{array}{l}\omega_{0}=0.15, \omega_{1}=1.00, \omega_{2}=5.39 \\
p_{0}=0.47, p_{1}=0.50, p_{2}=0.03\end{array}$ & -29884.7 & $\begin{array}{c}M 2 \text { vs. M1 } \\
2 \Delta \mathrm{LnL}=45 \\
\mathrm{df}=2, p=0.00\end{array}$ & $\underline{68}, \underline{116}, \underline{\mathbf{3 1 1}}, \underline{500}$ \\
\hline & M3: discrete & $\begin{array}{l}\omega_{0}=0.04, \omega_{1}=0.33, \omega_{2}=1.02 \\
p_{0}=0.26, p_{1}=0.52, p_{2}=0.22\end{array}$ & -29726.0 & $\begin{array}{c}\text { M3 vs. M0 } \\
2 \Delta \mathrm{LnL}=1614 \\
\mathrm{df}=4, p=0.00\end{array}$ & $\begin{array}{l}1, \mathbf{2 0}, \mathbf{4 6}, \mathbf{6 3}, \mathbf{6 8}, \mathbf{7 3}, \mathbf{8 9}, \mathbf{9 1}, 92, \underline{\mathbf{9 6},} \\
\mathbf{9 7}, \underline{\mathbf{1 1 6}}, 139, \mathbf{1 6 1}, \mathbf{1 7 6}, 190,204, \underline{208}, \\
212, \mathbf{2 2 3}, \mathbf{2 3 8}, 241,263, \mathbf{2 6 7}, \mathbf{2 8 6}, \\
\mathbf{2 8 7}, 299, \mathbf{3 0 6}, \underline{\mathbf{3 1 1}}, 319, \mathbf{3 3 3}, \mathbf{3 3 8}, \\
\mathbf{3 7 3}, \underline{\mathbf{4 0 5}}, \mathbf{4 1 3}, \mathbf{4 7 0}, \mathbf{4 8 1}, \mathbf{4 8 3}, \underline{\mathbf{5 0 0}}, \\
522, \mathbf{5 3 1}, \underline{\mathbf{5 3 4}}, \underline{\mathbf{5 4 4}}, \mathbf{5 9 3}, \underline{\mathbf{5 9 7}}, \mathbf{6 6 0}\end{array}$ \\
\hline & M7: $\beta$ & $p=0.66, q=1.06$ & -29714.7 & & Not allowed \\
\hline & M8: $\beta+\omega S>1$ & $\begin{array}{c}p=0.71, q=1.24 \\
\quad \omega=2.80 \\
p_{0}=0.96, p_{1}=0.04\end{array}$ & -29694.4 & $\begin{array}{c}\mathrm{M} 8 \text { vs. M7 } \\
2 \Delta \mathrm{LnL}=40.6 \\
\mathrm{df}=2, p=0.00\end{array}$ & $\underline{68}, \underline{116}, \underline{311}$ \\
\hline \multirow[t]{3}{*}{ Datamonkey ${ }^{c}$} & SLAC & & & & $\underline{68}, \underline{129}, \underline{534}$ \\
\hline & FEL & & & & $\begin{array}{l}\frac{68}{463}, \underline{96}, \underline{129}, 152, \underline{208}, 347,371, \underline{405} \\
655, \underline{789}, \underline{844}, \underline{44}, 569, \underline{597}, 639\end{array}$ \\
\hline & REL & & & & $702, \underline{789} 823,834, \underline{844}, 853$ \\
\hline
\end{tabular}

${ }^{a}$ Codons identified by more than one maximum likelihood method are underlined.

${ }^{b}$ Only positively selected sites with Bayesian posterior probabilities equal or $>95 \%$ are indicated. Sites with a posterior probability $>99 \%$ are highlighted in bold.

${ }^{c}$ Only positively selected sites with $p<0.01$ (SLAC and FEL) or Bayes factor $>50$ (REL) are shown.

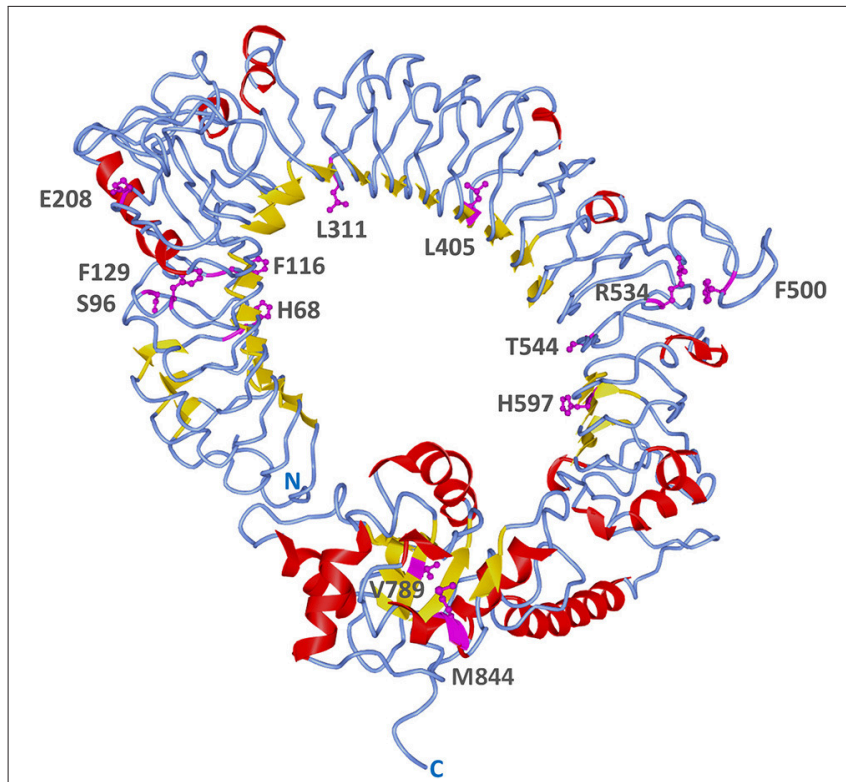

FIGURE 7 | Positively selected sites on B. pectinirostris TIr23a. Its tertiary structure was obtained by homology modeling using human TLR5 (PDB 3JOA) as template. Sites found to be under positive selection by more than one likelihood model are indicated in magenta and their side chains are shown. Residue numbers are based on the codon alignment used for positive selection analysis (Supplementary Figure 5).

followed by significantly dropping down for the rest experimental period (Figure 12B). Among tlr23 paralogues, several genes were significantly down-regulated by LPS stimulation in both spleen and kidney (Figures 9, 10). However, the expression profiles of these genes didn't show time-dependent significant differences. Similar expression patterns were observed in response profiles of $t l r 23 c$ and $t l r 23 g$ after poly(I:C) stimulation in the kidney (Figures 12H,L). In spleen, except $t \operatorname{lr} 23 c$ and $t$ lr23f showed similar down-regulation patterns (Figures $1 \mathbf{1 H}, \mathbf{K}$ ), tlr23b, tlr23e, and $t \operatorname{lr} 23 g$ were significantly stimulated by poly(I:C) at 3 or 12 hpi (Figures 11G,J,L). However, the expression profile of tlr23b showed significant increasing trend during sampling periods in both control and treated groups (Figure 11G). In contrast to tlr23g (Figure 11L), only tlr23e showed a clear significantly timedependent increase pattern in the spleen, and reached the highest level at 24 hpi (Figure 11J).

\section{DISCUSSION}

Several types of gene duplication have been observed in genome to date, including whole genome duplication (43), segmental duplication (44), DNA-mediated duplicative transposition and retrotransposition (45). Paralogous or duplicated Tlr genes in teleosts, probably resulting from the third or fourth round of whole genome duplication event, have been identified in Danio rerio (46, 47), Oncorhynchus mykiss (48), and Cyprinus carpio (49). In the present study, we reported an extensive duplication of $\operatorname{tl} 23$ genes (7 paralogues). Previous study in Atlantic cod has reported an extensive duplication of tra2 genes (12 paralogues) (13). Such intensive duplication may be not only due to genome duplication but also due to other mechanisms of gene duplication. DNA-mediated duplicative transposition and retrotransposed duplication are ongoing 
A

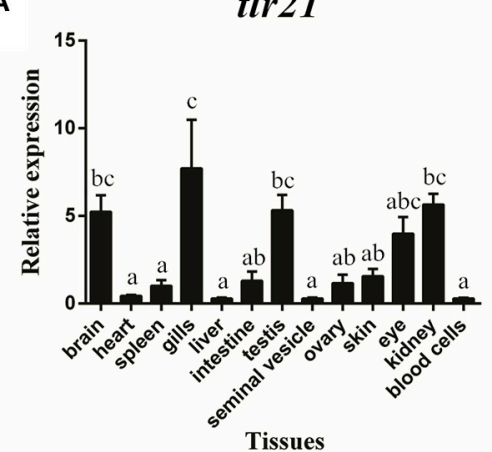

D

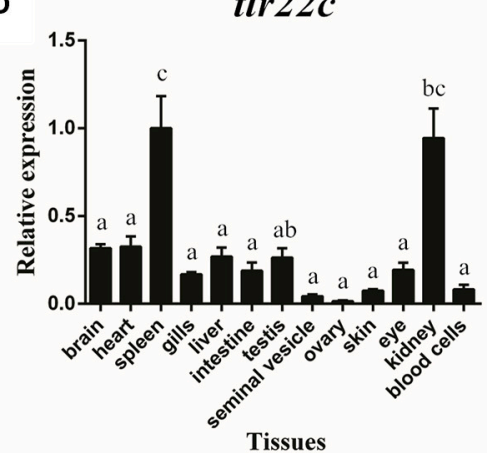

G
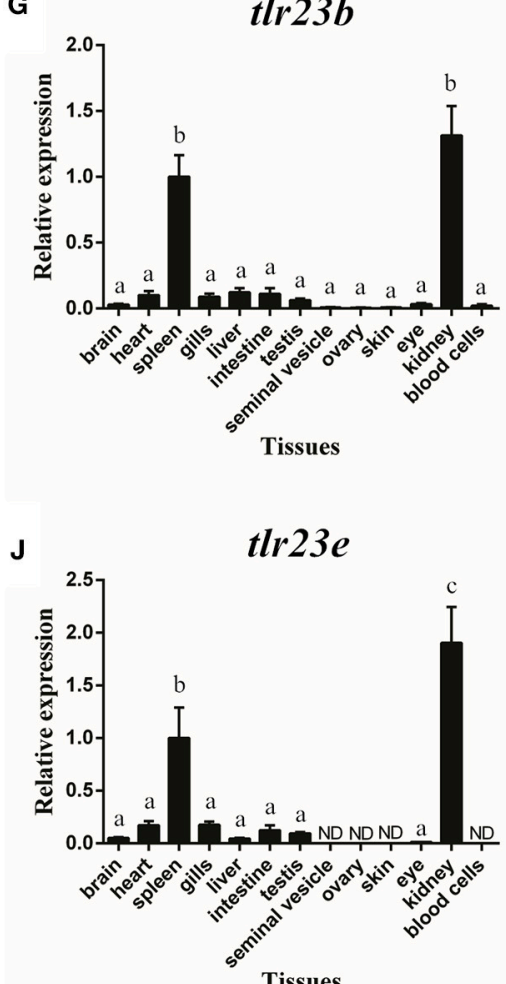

B

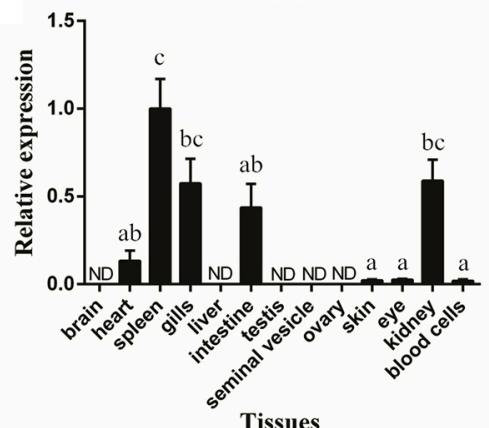

Tissues

E

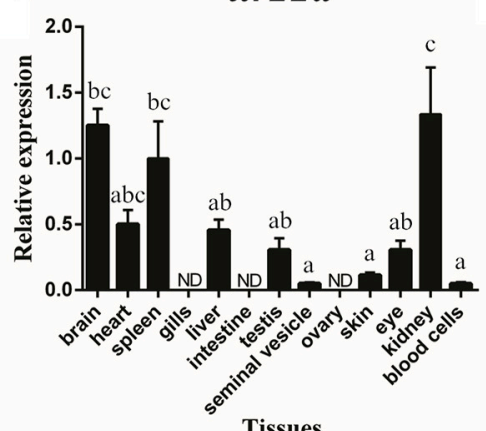

Tissues

H

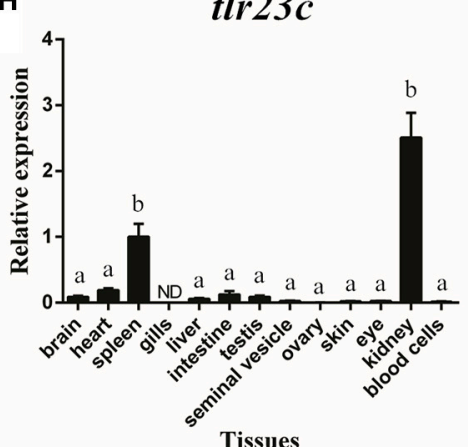

K

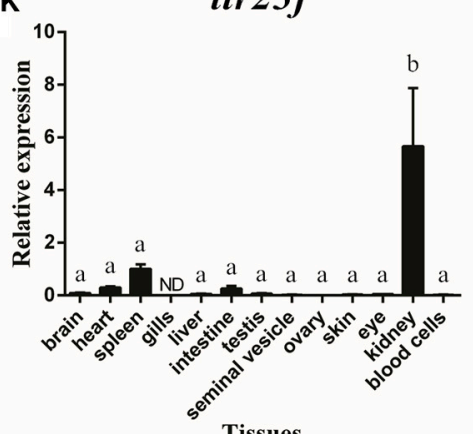

C

$\operatorname{tlr} 22 b$

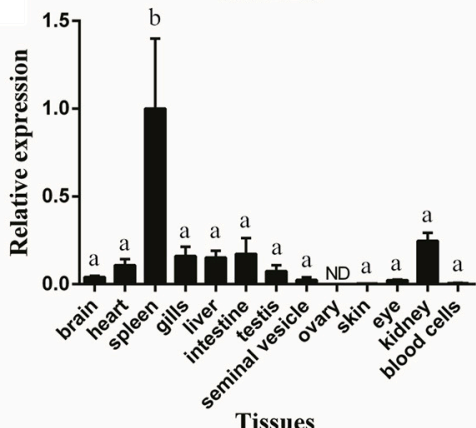

Tissues

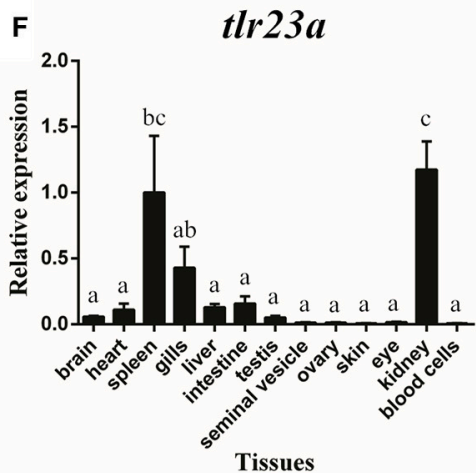

Tissues

tlr23d
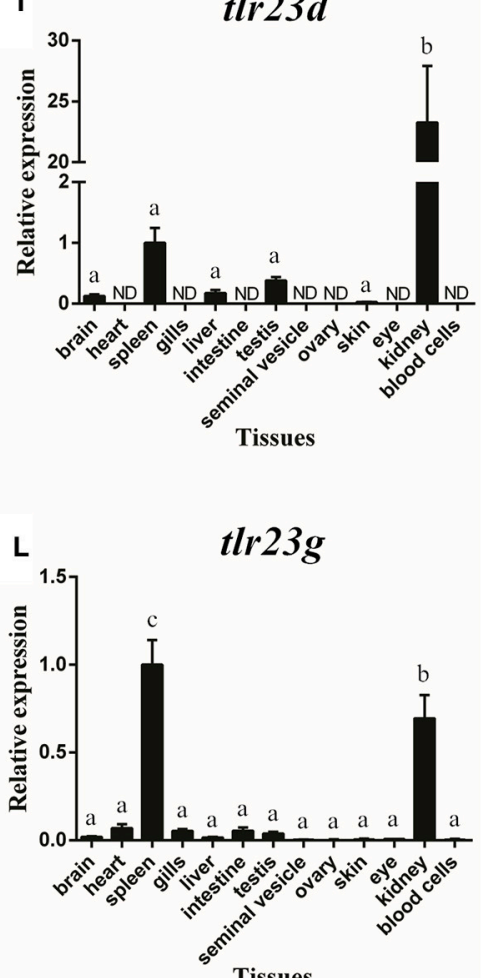

FIGURE 8 | Tissue distribution of t/r21 (A), t/r22 (B-E), and t/r23 (F-L) paralogues in B. pectinirostris. The levels of the respective mRNAs were determined using qPCR and normalized to the internal housekeeping gene eef1 $\alpha$. The results were expressed as mean \pm SEM ( $n=7)$. Columns with different letters are significantly differences with each other ( $p<0.05$, One-way ANOVA followed by Tukey's test). 


\section{Spleen $\square$ PBS}

A

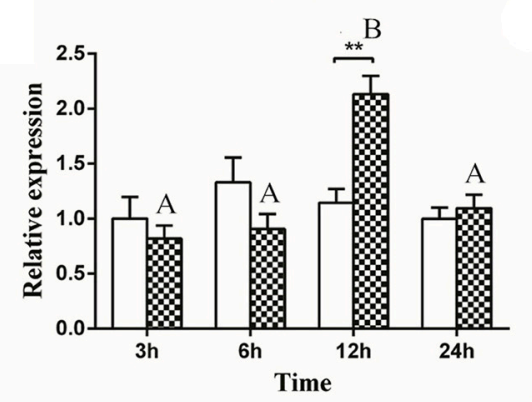

D

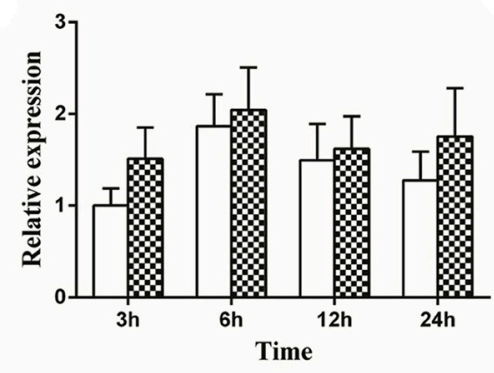

G

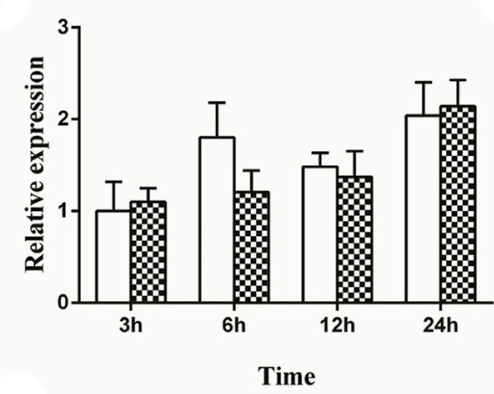

tlr23e

J

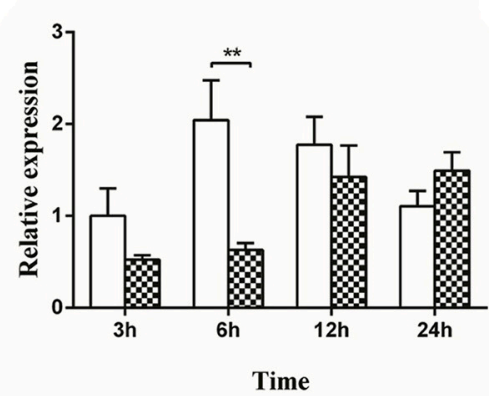

B

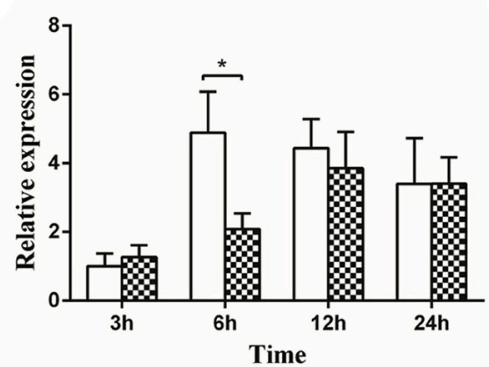

E

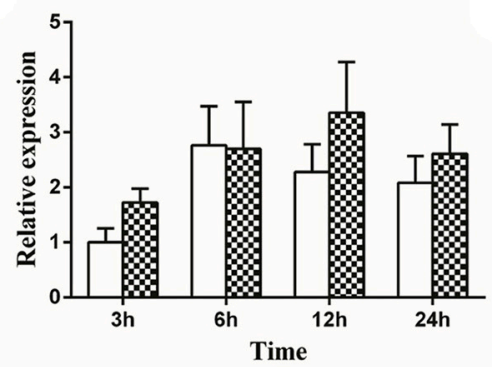

tlr23c

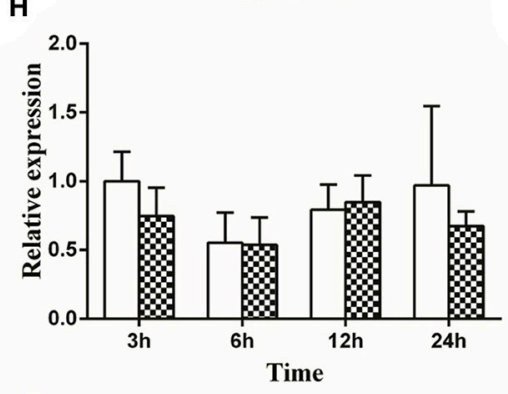

$\operatorname{tr} 23 f$

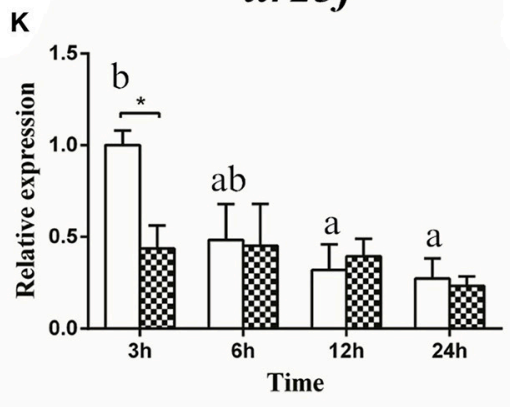

c

$t \operatorname{tr} 22 b$

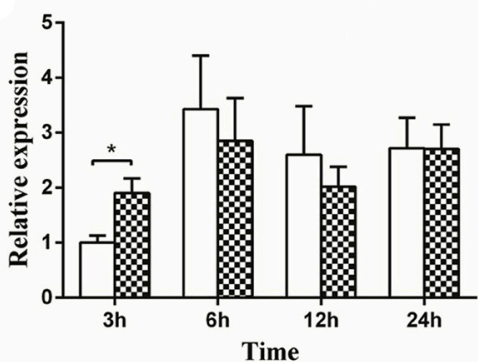

tlr23a

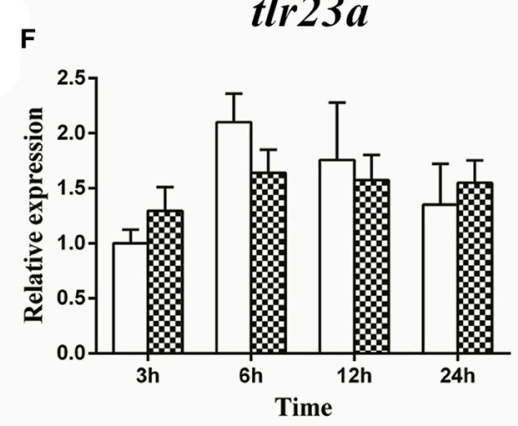

tlr23d

I
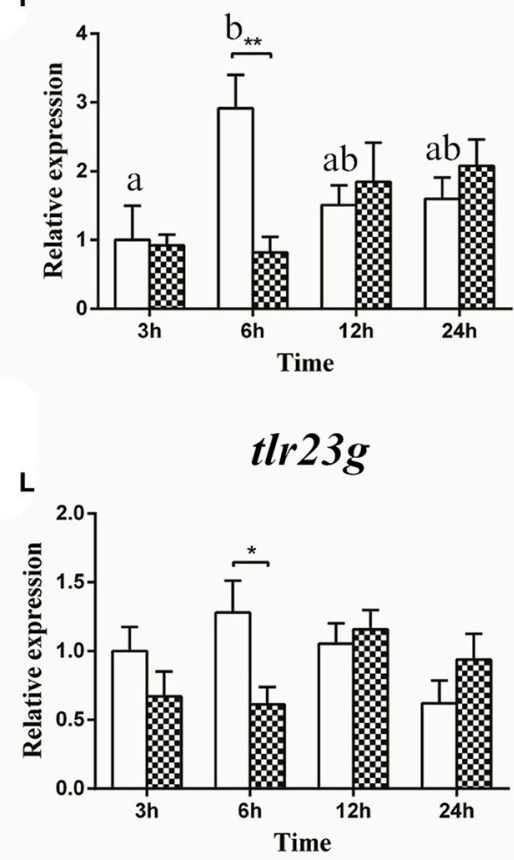

FIGURE 9 | The relative expression of t/r21 (A), t/r22 (B-E), and t/r23 (F-L) paralogues in the spleen after intraperitoneal injection with LPS. Relative expression of these $12 \mathrm{t}$ tr genes in the kidney were examined at different time points $(3,6,12,24 \mathrm{~h}$ ) by Real-time qPCR and all data were expressed as the mean \pm SEM $(n \geq 5)$ and normalized to the expression of eef1 $\alpha$. Significant difference between PBS and LPS treated group was indicated with * $(p<0.05$, Student's $t$-test) or ** $(p<0.01$, Student's $t$-test). Columns with different letters are significantly differences with each other ( $p<0.05$, One-way ANOVA followed by Tukey's test). Lowercase and uppercase letters indicate control and treated groups, respectively. 


\section{Kidney $\square$ PBS}
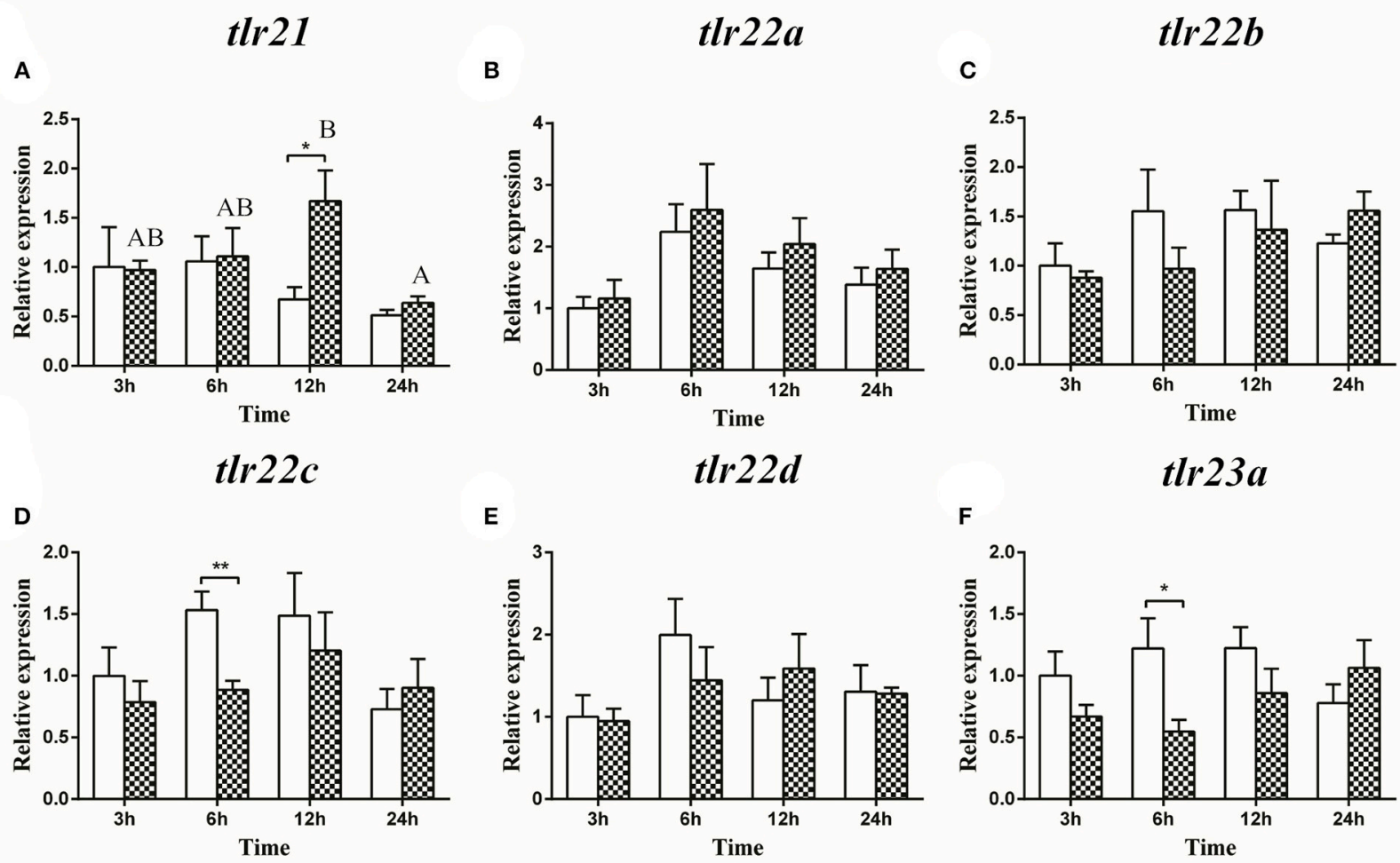

tlr22d

E

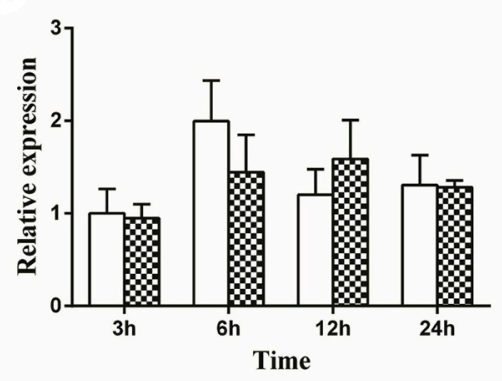

tIr23a

F

G

tlr23b

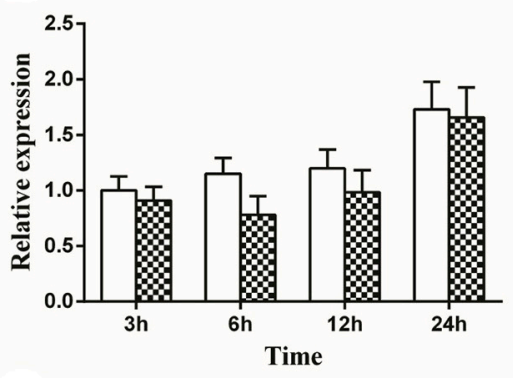

H

tlr23c
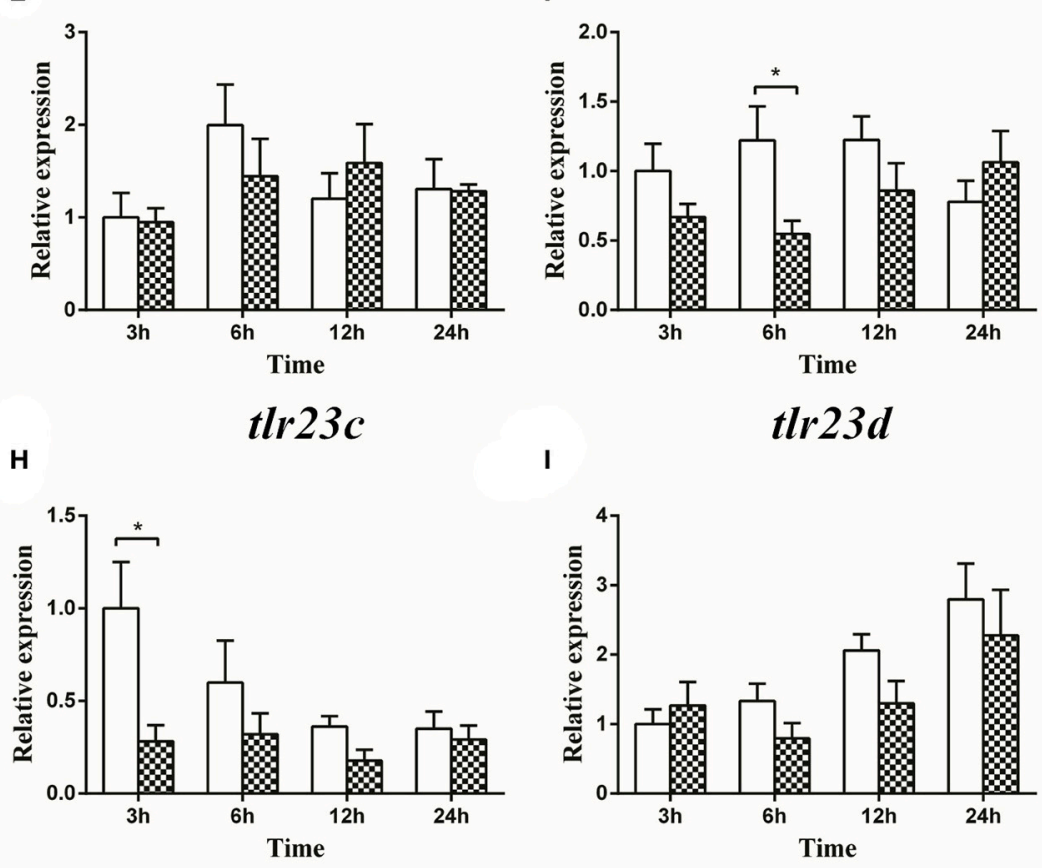

tlr23d

J

tlr23e
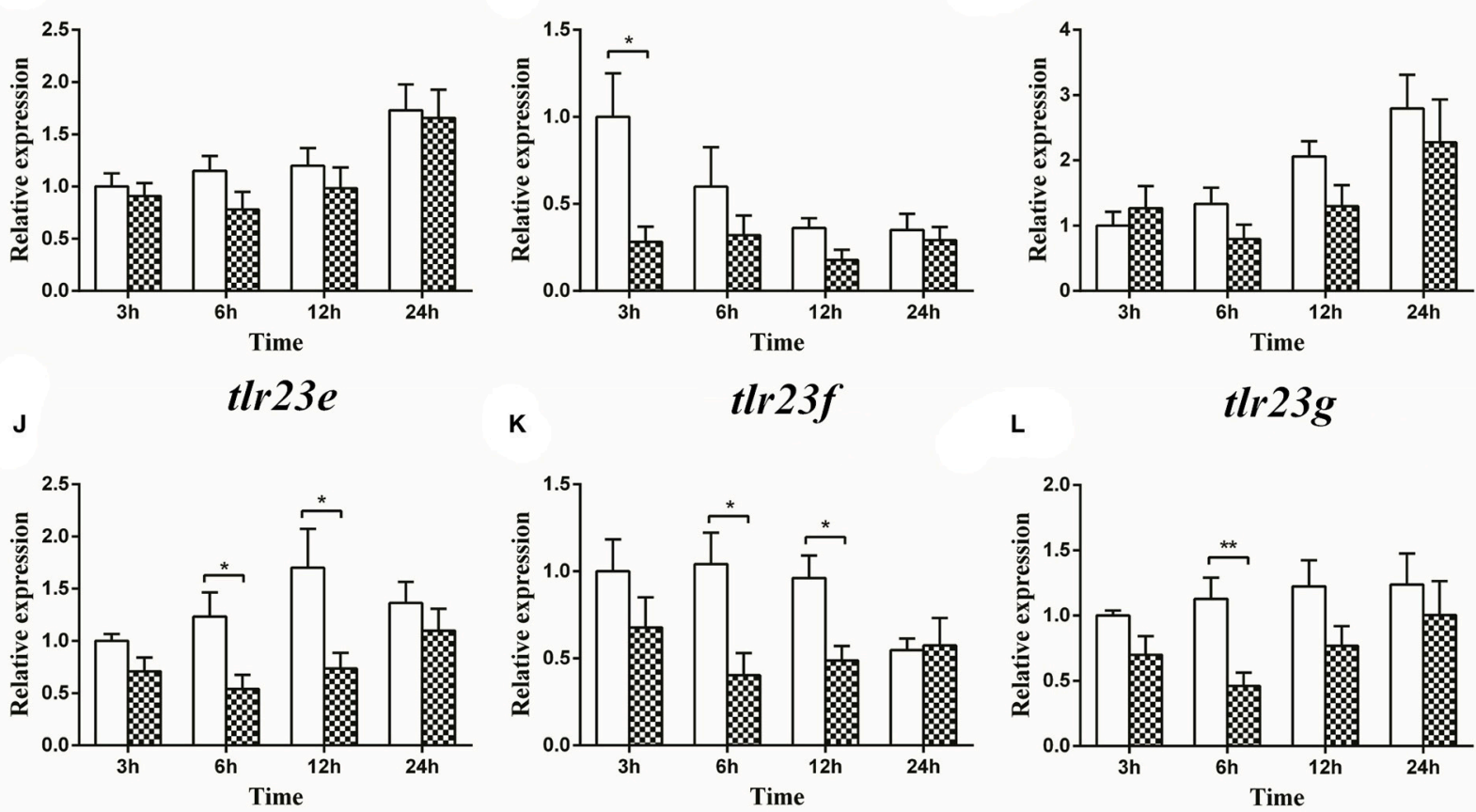

ᄂ $\quad$ tlr $23 g$

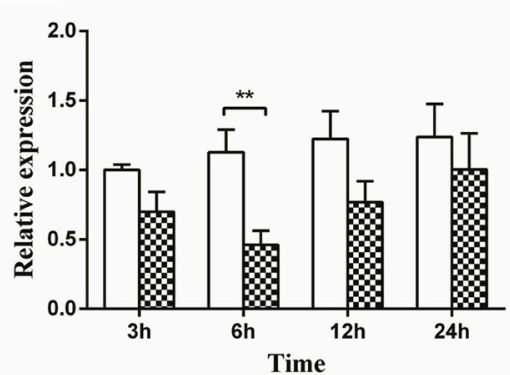

FIGURE 10 | The relative expression of tr21 (A), tr22 (B-E), and t/r23 (F-L) paralogues in the kidney after intraperitoneal injection with LPS. Relative expression of these 12 tir genes in the kidney were examined at different time points $(3,6,12,24 \mathrm{~h})$ by Real-time GPCR and all data were expressed as the mean \pm SEM $(n \geq 5)$ and normalized to the expression of eef1 $\alpha$. Significant difference between PBS and LPS treated group was indicated with * $p<0.05$, Student's $t$-test) or ** $p<0.01$, Student's $t$-test). Columns with different letters are significantly differences with each other $(p<0.05$, One-way ANOVA followed by Tukey's test). 


\section{Spleen $\square$ poly(I:C)}
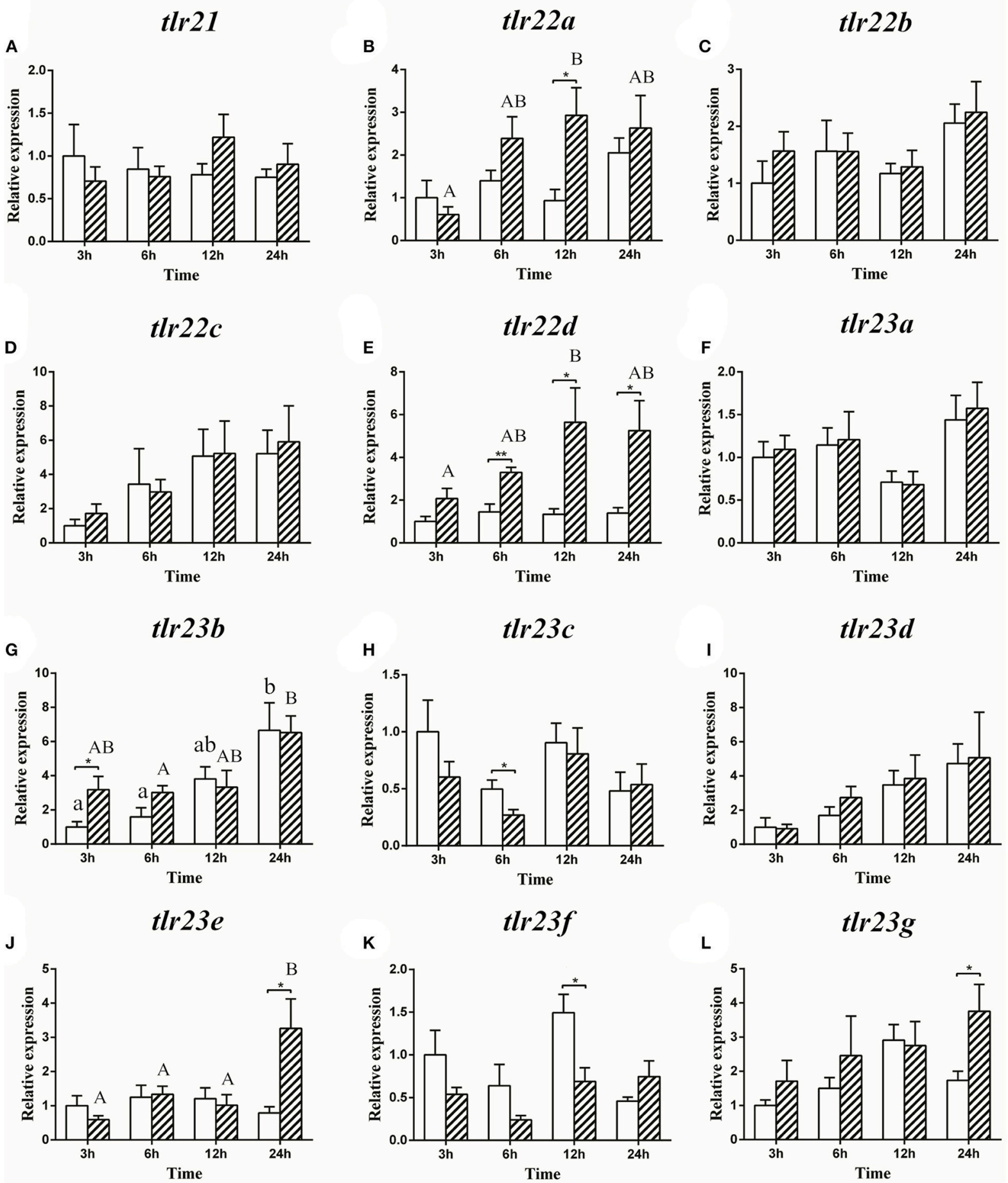

FIGURE 11 | The relative expression of tIr21 (A), trr22 (B-E), and tIr23 (F-L) paralogues in the spleen after intraperitoneal injection with poly(I:C). Relative expression of these $12 \mathrm{t}$ Ir genes in the kidney were examined at different time points $(3,6,12,24 \mathrm{~h})$ by Real-time qPCR and all data were expressed as the mean \pm SEM $(n \geq 5)$ and normalized to the expression of eef1 $\alpha$. Significant difference between PBS and poly(l:C) treated group was indicated with * $p<0.05$, Student's $t$-test) or ** $p<0.01$, Student's $t$-test). Columns with different letters are significantly differences with each other ( $p<0.05$, One-way ANOVA followed by Tukey's test). Lowercase and uppercase letters indicate control and treated groups, respectively. 


\section{Kidney $\underset{\mathbb{P} \text { Poly(I:C) }}{\square \text { PBS }}$}
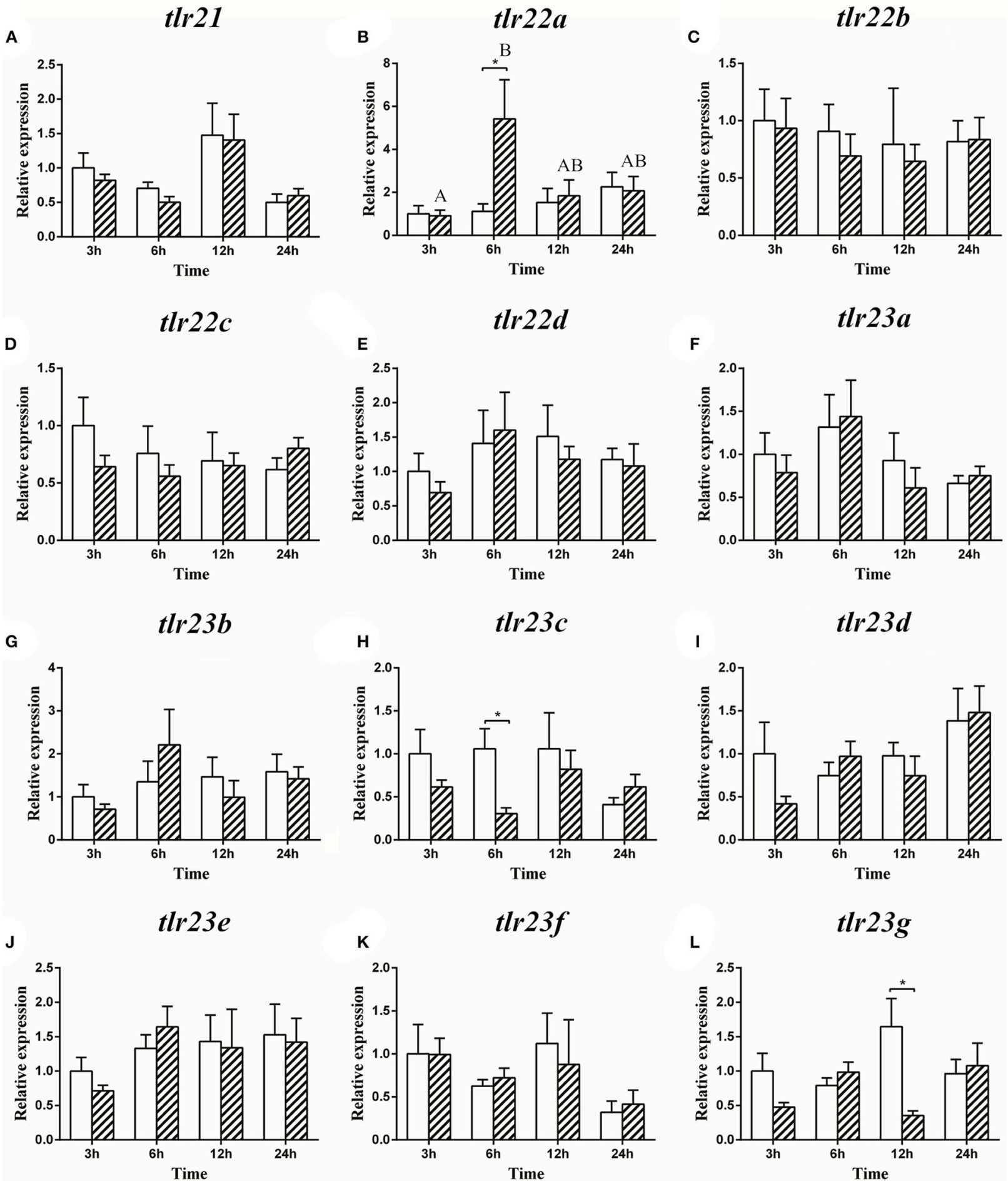

FIGURE 12 | The relative expression of t/r21 (A), t/r22 (B-E), and t/r23 (F-L) paralogues in the kidney after intraperitoneal injection with poly(I:C). Relative expression of these $12 \mathrm{t}$ Ir genes in the kidney were examined at different time points $(3,6,12,24 \mathrm{~h}$ ) by Real-time qPCR and all data were expressed as the mean \pm SEM ( $n \geq 5)$ and normalized to the expression of eef1 $\alpha$. Significant difference between PBS and poly(l:C) treated group was indicated with * $(p<0.05$, Student's $t$-test). Columns with different letters are significantly differences with each other ( $p<0.05$, One-way ANOVA followed by Tukey's test). 
process, continually expanding the genetic repertoire of modern organisms (45). A common result of DNA-mediated duplication is a new gene that preserves the intron-exon architecture and the cis-regulatory elements of the parental gene, whereas retrotransposed duplication often generates an intronless gene copy as a result of a process in which a spliced mRNA is reversetranscribed into cDNA and spontaneously integrated into a random genomic location (45). Besides, it has been suggested that the influence of short tandem repeats may substantially increase the rate of duplication of a DNA segment located between them (50). In B. pectinirostris, both $t$ tr22 and tlr23 paralogues showed similar intron-exon architectures. Moreover, short tandem repeat sequences were identified in the UTRs of $t \operatorname{lr} 22 b, t \operatorname{lr} 22 d, t \operatorname{lr} 23 a$, and $t \operatorname{lr} 23 d$. Thus, it is possible that tlr22 and tlr23 paralogues resulted from the DNA-mediated duplicative transposition.

Similar to other teleosts $(13,51,52)$, there exists only one tlr21 ortholog encoded by a single exon in B. pectinirostris. Besides, in B. pectinirostris and other fish species, Tlr21 molecules do not have an LRR-CT, in contrast to the chicken and goose TLR21 sequences (51). The structure of these molecules may suggest that the function of Tlr21 is highly conserved in teleosts. B. pectinirostris tlr21 (Bptlr21) was constitutively expressed at different levels in all examined tissues, which is similar to the expression patterns of tlr21 in large yellow croaker (L. crocea) and Atlantic cod $(13,51)$. TLR21 in chicken (G. gallus) and zebrafish play a role in immune response to bacterial infection by recognizing CpG-oligodeoxynucletides (CpG-ODNs) as a danger signal $(53,54)$. In the present study, upon stimulation with LPS, the expression of Bptlr21 was significantly up-regulated in the spleen and kidney at $12 \mathrm{hpi}$ in B. pectinirostris. In the mammal immune system, the complex of TLR4, CD14, and MD2 has been proved to be the receptor for LPS at the cell surface (55). Unlike in mammals, trr gene has been lost from the genomes of most fishes (9) including B. pectinirostris, and Tlr4 in fish does not recognize the stimulation of LPS (56). However, LPS does have multiple biological effects on fish including enhancing the production of immune related cytokines $(57,58)$. Recently, study in miiuy croaker (Miichthys miiuy) showed that NOD1 can identify LPS and activate the NF- $\mathrm{KB}$ signal pathway by recruiting RIPK2 and then promoting the expression of inflammatory cytokines to induce the resistance of organism against bacterial infection (59). Another study further demonstrated that scavenger receptor class B 2a (SRB2a), a novel isoform of the mammalian SRB2 gene, mediates LPS internalization for interaction with NOD1 and NOD2 to initiate NF-KB in teleost macrophages (60). The results from the present study suggest that BpTlr21 may be involved in immune response to bacterial infection in B. pectinirostris, and further studies should be focused on whether the stimulatory effects of LPS on Bptlr21 was mediated by SRB2a and NOD in B. pectinirostris.

The four $t$ tr22 orthologs cloned from B. pectinirostris grouped under a single clade in the phylogenetic tree, separately from the Atlantic cod orthologs of Bptlr22, suggesting that the expansion of tlr22 occurred independently during evolution of these two species. Bptlr22 orthologs are not adjacent in the B. pectinirostris genome. The genomic region surrounding thr22a is conserved in comparison with tlr22 from several fish species, and the region contains the genes $s h 3 k b p 1, m a p 3 k 15$, and $c n k s r 2$. It has been proposed that selection favors the organization of gene clusters to facilitate the coordinated control of gene expression and related biological processes (61). In mammals, Sh3kbp1 is involved in the B cell receptor signaling in normal lymphocytes (62). Besides, it has been reported that the B-cell response to CpG S-ODN is mediated through TLR9 (63). Therefore, conserved syntenic localization between Tlr22a and Sh3kbp1 suggests that Tlr22a may participate in biological processes related to B cells in teleosts. Map3k15 (ASK3) is likely a component of ASK1 (Map3k5) signalosome and can interact with ASK1 (64), and ROS-dependent activation of TRAF6-ASK1p38 pathway is crucial for TLR4-mediated innate immunity (65), which suggest that Map3k15 may take part in Tlr22a signaling pathway. Further study would be interesting to examine these adjacent genes involved in the Tlr22a mediated signaling pathway in B. pectinirostris.

In other teleosts, poly(I:C) challenge up-regulated the expression of $t \operatorname{tr} 22$ in many tissues $(66,67)$. It was reported, in tiger pufferfish, that Tlr22 localizes to the cell surface and recognizes long-sized dsRNA or poly(I:C) and links the IFNinducing pathway via the TRIF adaptor (68). In the present study, the expression of tlr22a and tlr22d in spleen, and tlr22a in kidney showed clear time-dependent up-regulation after poly(I:C) stimulation. These results may suggest that the function of Tlr22a and Tlr22d might play a role in innate immune response to virus. Nevertheless, some Bptlr22 paralogues (including tlr22a) showed a response after LPS stimulation. A study in Atlantic cod indicated that most of $t$ lr 22 orthologs transcripts are up-regulated after bacterial bath challenge (13). However, it is worth noting that, after LPS stimulation, the expression profiles of Bptlr22 paralogues didn't show time-dependent significant differences. Therefore, further studies would be necessary to investigate the possibility that the expansion of Tlr22 likely increases the detectable ligand repertoire, e.g., to recognize dsRNA and PAMPs from pathogen origin.

The function of TLR23 is still largely unknown (13). $B$. pectinirostris encoded 7 copies of $t$ tr 23 genes and possessed the largest group of Tlr23 in vertebrates sequenced. It is noteworthy that all BpTlr23 except Tlr23a clustered under a single clade in phylogenetic tree, which suggests that BpTlr23 under the same clade may evolve independently in comparison with BpTlr23a and other teleost Tlr23. We found that Tlr23a genes in several fish species are often adjacent to the ppme1-like gene, which suggests that the function of tr $23 a$ may be conserved in different teleost species. Recently, it was found that activation of TLR4 signaling pathway will increase the expression of MFHAS1, which further inhibits expression of inflammatory factors and plays a role in negatively regulating TLR4 signaling pathway (69). During this process, MFHAS1 combines with the B and C subunits of PP2A, which involves up-regulation of PPME-1 (70). Therefore, it is possible that ppme1 may be involved in the negative regulation of signaling pathway of $t \operatorname{lr} 23 a$, and even other tlr23 paralogues.

Tlr23 paralogues in B. pectinirostris responded differently to LPS and poly(I:C) challenges. Tlr23e and $t \operatorname{lr} 23 g$ were significantly up-regulated in the spleen upon poly(I:C) stimulation, which 
suggest that $t l r 23 e$ and $t l r 23 g$ may participate in antiviral immune processes. In contrast to up-regulation, several B. pectinirostris tlr23 paralogues showed down-regulation after LPS and poly(I:C) challenges. Atlantic cod thr23a is also significantly reduced upon a bath challenge with Gram-negative bacteria $V$. anguillarum (13). In teleost fish, several studies have identified subsets of microRNAs (miRNAs) that are differentially expressed in organs challenged with DNA or RNA virus, LPS or poly (I:C) (71). Based on the use of bioinformatics approaches and whole transcriptome analysis, increasing studies have discovered that miRNAs negatively regulate the expression of Tlr genes (72). It would be necessary to predict miRNA regulators of th 23 paralogues and elucidate their roles in the regulation of tr 23 paralogues in B. pectinirostris.

The average number of synonymous changes was higher than the non-synonymous substitutions in all pairwise comparisons between th11 genes in B. pectinirostris, indicating overall purifying selection, likely due to functional constraints (73). In fish, the prevalence of purifying selection signatures has been reported not only in tlr genes (13) but also in other immunerelated genes, such as antimicrobial peptides (39). Nevertheless, substitution rates were not uniform across $\operatorname{tr} 11$ paralogues in $B$. pectinirostris and the observed differences contribute to explaining how the TIR domains of Tlr21, Tlr22, and Tlr23 are more conserved than their LRR regions. The TIR domain is generally conserved across species as well as between different TLRs, since it is involved in signal transduction (74).

Homology modeling of $B$. pectinirostris Tlr23a based on human TLR5 revealed a characteristic horseshoe-shaped structure with a single ectodomain architecture. Most positively selected sites were found in the ectodomain, especially in the convex side of the extracellular solenoid structure, which is most important for ligand binding. Non-synonymous substitutions at the positively selected sites may affect ligand specificity through changes in the amino acids within the beta sheets or in the convex surface of the horseshoe-shaped domain (75). For example, variations in the LRR coil at position 68 between small polar ( $S$ and $\mathrm{T})$, positively charged $(\mathrm{R}, \mathrm{K}$ and $\mathrm{H})$ and large hydrophobic

\section{REFERENCES}

1. Kawasaki T, Kawai T. Toll-like receptor signaling pathways. Front Immunol. (2014) 5:461. doi: 10.3389/fimmu.2014.00461

2. Janeway CA. Approaching the Asymptote? Evolution and revolution in immunology. SH Symp Quant Biol. (1989) 54:1-13. doi: 10.1101/SQB.1989.054.01.003

3. Moresco EM, Lavine D, Beutler B. Toll-like receptors. CSH Symp Quant Biol. (2011) 21:R488-93. doi: 10.1016/j.cub.2011.05.039

4. Anderson KV, Bokla L, Nüssleinvolhard C. Establishment of dorsal-ventral polarity in the Drosophila embryo: the induction of polarity by the Toll gene product. Cell. (1985) 42:791-8. doi: 10.1016/0092-8674(85)90275-2

5. Lemaitre B, Nicolas E, Michaut L, Reichhart JM, Hoffmann JA. The dorsoventral regulatory gene cassette spätzle/Toll/cactus controls the potent antifungal response in Drosophila adults. Cell. (1996) 86:973-83. doi: 10.1016/S0092-8674(00)80172-5

6. Nie L, Cai SY, Shao JZ, Chen J. Toll-like receptors, associated biological roles, and signaling networks in non-mammals. Front Immunol. (2018) 9:1523. doi: 10.3389/fimmu.2018.01523 aromatic ( $\mathrm{F}$ and $\mathrm{W}$ ) amino acids will likely affect the polarity and structure of the ectodomain, thus affecting ligand specificity. This functional diversification of the B. pectinirostris Tlr11 family through positive selection may be linked to adaptation to evolving pathogens.

In conclusion, we identified and annotated $12 \mathrm{tlr}$ genes (one tlr21, 4 tlr22, and 7 tr 23) representing all members of the high expanded Tlr11 family in the mudskipper B. pectinirostris. The expanded Tlr11 family in B. pectinirostris provides a good model to better understand how and why so many TLR genes have been retained during vertebrate evolution.

\section{AUTHOR CONTRIBUTIONS}

HQ was involved in entire study. JF analyzed the positive selection. XY, HW, and HY performed the partial synteny and chromosome location analyses. YZ, SH, DL, and QW analyzed results. SC and WH conceived and supervised the project, analyzed results and prepared the manuscript.

\section{FUNDING}

This research was supported by the National Natural Science Foundation of China (No. 31672628), Fundamental Research Funds for the Central Universities (No. 20720180123), Program for New Century Excellent Talents in Fujian Province University, Shenzhen Special Program for Development of Emerging Strategic Industries (No. JSGG20170412153411369), Shenzhen Dapeng Special Program for Industrial Development (No. KY20170204), Shenzhen Special Program for Development of Strategic Emerging and Future Industries (No. 20170428173357698).

\section{SUPPLEMENTARY MATERIAL}

The Supplementary Material for this article can be found online at: https://www.frontiersin.org/articles/10.3389/fimmu. 2019.00343/full\#supplementary-material

7. Palti Y. Toll-like receptors in bony fish: from genomics to function. Dev Comp Immunol. (2011) 35:1263-72. doi: 10.1016/j.dci.2011.03.006

8. Ji J, Rao Y, Wan Q, Liao Z, Su J. Teleost-specific TLR19 localizes to endosome, recognizes dsRNA, Recruits TRIF, Triggers both IFN and NF- $\kappa$ B pathways, and protects cells from grass carp reovirus infection. J Immunol. (2018) 200:573. doi: 10.4049/jimmunol.1701149

9. Rauta PR, Samanta M, Dash HR, Nayak B, Das S. Toll-like receptors (TLRs) in aquatic animals: signaling pathways, expressions and immune responses. Immunol Lett. (2014) 158:14-24. doi: 10.1016/j.imlet.2013. 11.013

10. Wang Y, Li J, Han J, Shu C, Xu T. Identification and characteristic analysis of TLR28: A novel member of the TLR1 family in teleost. Dev Comp Immunol. (2016) 62:102-7. doi: 10.1016/j.dci.2016.05.001

11. Zhang X, Zhang G, Shi Z, Yuan Y, Zheng H, Lin L, et al. Expression analysis of nine Toll-like receptors in yellow catfish (Pelteobagrus fulvidraco) responding to Aeromonas hydrophila challenge. Fish Shellfish Immun. (2017) 63:384-93. doi: 10.1016/j.fsi.2017.02.021

12. Sundaram AY, Consuegra S, Kiron V, Fernandes JM. Positive selection pressure within teleost Toll-like receptors $t$ lr21 and $t$ lr22 subfamilies and their 
response to temperature stress and microbial components in zebrafish. Mol Biol Rep. (2012) 39:8965-75. doi: 10.1007/s11033-012-1765-y

13. Sundaram AY, Kiron V, Dopazo J, Fernandes JM. Diversification of the expanded teleost-specific toll-like receptor family in Atlantic cod, Gadus morhua. BMC Evol Biol. (2012) 12:256. doi: 10.1186/1471-2148-12-256

14. Qi D, Xia M, Chao Y, Zhao Y, Wu R. Identification, molecular evolution of toll-like receptors in a Tibetan schizothoracine fish (Gymnocypris eckloni) and their expression profiles in response to acute hypoxia. Fish Shellfish Immun. (2017) 68:102-13. doi: 10.1016/j.fsi.2017.07.014

15. Wang J, Zhang Z, Fu H, Zhang S, Liu J, Chang F, et al. Structural and evolutionary characteristics of fish-specific TLR19. Fish Shellfish Immun. (2015) 47:271-9. doi: 10.1016/j.fsi.2015.09.005

16. Han C, Li Q, Zhang Z, Huang J. Characterization, expression, and evolutionary analysis of new TLR3 and TLR5M genes cloned from the spiny eel Mastacembelus armatus. Dev Comp Immunol. (2017) 77:174-87. doi: 10.1016/j.dci.2017.08.007

17. Varriale S, Ferraresso S, Giacomelli S, Coscia MR, Bargelloni L, Oreste U. Evolutionary analysis of Antarctic teleost Toll-like receptor 2. Fish Shellfish Immun. (2012) 33:1076-85. doi: 10.1016/j.fsi.2012.07.010

18. Chen JS, Wang TY, Tzeng TD, Wang CY, Wang D. Evidence for positive selection in the TLR9 gene of teleosts. Fish Shellfish Immun. (2008) 24:234-42. doi: 10.1016/j.fsi.2007.11.005

19. Hong W, Chen S, Zhang Q, Wang Q. Reproductive ecology of the mudskipper Boleophthalmus pectinirostris. ACTA Oceanol Sin. (2007) 26:72-81.

20. Morii H, Nishikata K, Tamura O. Nitrogen excretion of mudskipper fish Periophthalmus cantonensis and Boleophthalmus pectinirostris in water and on land. Comp Biochem Physiol A. (1978) 60:189-93. doi: 10.1016/0300-9629(78)90229-3

21. Chen S, Hong W, Zhang Q, Su Y. Why does the mudskipper Boleophthalmus pectinirostris form territories in farming ponds? J Mar Biol Assoc UK. (2007) 87:615-9. doi: 10.1017/S0025315407054434

22. Chen S, Hong W, Zhang Q, Wu R, Wang Q. Rates of oxygen consumption and tolerance of hypoxia and desiccation in Chinese black sleeper (Bostrichthys sinensis) and mudskipper (Boleophthalmus pectinirostris) embryos. Acta Oceanol Sin. (2006) 25:91-8.

23. Yokoya S, Tamura OS. Fine structure of the skin of the amphibious fishes, Boleophthalmus pectinirostris and Periophthalmus cantonensis, with special reference to the location of blood vessels. J Morphol. (2010) 214:287-97. doi: 10.1002/jmor.1052140305

24. Li Z, Hong WS, Qiu HT, Zhang YT, Yang MS, You XX, et al. Cloning and expression of two hepcidin genes in the mudskipper (Boleophthalmus pectinirostris) provides insights into their roles in male reproductive immunity. Fish Shellfish Immun. (2016) 56:239-47. doi: 10.1016/j.fsi.2016.07.025

25. Hong LY, Hong WS, Zhu WB, Shi Q, You XX, Chen SX. Cloning and expression of melatonin receptors in the mudskipper Boleophthalmus pectinirostris: their role in synchronizing its semilunar spawning rhythm. Gen Comp Endocr. (2014) 195:138-50. doi: 10.1016/j.ygcen.2013.11.004

26. Beachey EH, Giampapa CS, Abraham SN. Bacterial adherence: adhesin receptor-mediated attachment of pathogenic bacteria to mucosal surfaces. Am Rev Respir Dis. (1998) 138:45-8. doi: 10.1164/ajrccm/138.6_Pt_2.S45

27. Gomez D, Sunyer JO, Salinas I. The mucosal immune system of fish: the evolution of tolerating commensals while fighting pathogens. Fish Shellfish Immun. (2013) 35:1729-39. doi: 10.1016/j.fsi.2013. 09.032

28. You X, Bian C, Zan Q, Xu X, Liu X, Chen J, et al. Mudskipper genomes provide insights into the terrestrial adaptation of amphibious fishes. Nat Commun. (2014) 5:5594. doi: 10.1038/ncomms6594

29. Wang Y, Leung PC, Qian PY, Gu JD. Antibiotic resistance and plasmid profile of environmental isolates of Vibrio species from Mai Po Nature Reserve, Hong Kong. Ecotoxicology. (2006) 15:371-8. doi: 10.1007/s10646-006-0078-0

30. Pawar PR, Al-Tawaha ARM. Study of bacterial diversity in intertidal sediments along the marine ecosystem of Uran, Navi Mumbai. Adv Environ Biol. (2017) 11:25-38.

31. Alzohairy AM. BioEdit: an important software for molecular biology. Gerf Bull Biosci. (2011) 2:60-61.

32. Benson G. Tandem repeats finder: a program to analyze DNA sequences. Nucleic Acids Res. (1999) 27:573-80. doi: 10.1093/nar/27.2.573
33. Kumar S, Stecher G, Tamura K. MEGA7: molecular evolutionary genetics analysis version 7.0 for bigger datasets. Mol Biol Evol. (2016) 33:1870-4. doi: 10.1093/molbev/msw054

34. Nei M, Gojobori T. Simple methods for estimating the numbers of synonymous and nonsynonymous nucleotide substitutions. Mol Biol Evol. (1986) 3:418-26. doi: 10.1093/oxfordjournals.molbev.a040410

35. Yang Z, Nielsen R, Goldman N, Pedersen AM. Codon-substitution models for heterogeneous selection pressure at amino acid sites. Genetics. (2000) 155:431-49.

36. Nei M, Kumar S. Molecular Evolution and Phylogenetics. New York, NY: Oxford University Press (2000).

37. Yang Z. PAML 4: phylogenetic analysis by maximum likelihood. Mol Biol Evol. (2007) 24:1586-91. doi: 10.1093/molbev/msm088

38. Weaver S, Shank SD, Spielman SJ, Li M, Muse SV, Kosakovsky Pond SL. Datamonkey 2.0: a modern web application for characterizing selective and other evolutionary processes. Mol Biol Evol. (2018) 35:773-7. doi: $10.1093 / \mathrm{molbev} / \mathrm{msx} 335$

39. Fernandes JMO, Jareeporn R, Viswanath K. Atlantic cod piscidin and its diversification through positive selection. PLoS ONE. (2010) 5:e9501. doi: 10.1371/ journal.pone.0009501

40. Bienert S, Waterhouse A, de Beer TA, Tauriello G, Studer G, Bordoli L, et al. The swiss-model repository-new features and functionality. Nucleic Acids Res. (2017) 45:D313-9. doi: 10.1093/nar/gkw1132

41. Benkert $\mathrm{P}$, Biasini $\mathrm{M}, \mathrm{S}$ chwede T. Toward the estimation of the absolute quality of individual protein structure models. Bioinformatics. (2011) 27:343-50. doi: 10.1093/bioinformatics/btq662

42. Kenneth J, Livak TD. Analysis of relative gene expression data using realtime quantitative PCR and the $2^{-\Delta \Delta C T}$ method. Methods. (2001) 25:402-8. doi: $10.1006 /$ meth.2001.1262

43. Dehal P, Boore JL. Two rounds of whole genome duplication in the ancestral vertebrate genome. PLoS Biol. (2005) 3:e314. doi: 10.1371/journal.pbio.0030314

44. Sharp AJ, Locke DP, McGrath SD, Cheng Z, Bailey JA, Vallente RU, et al. Segmental duplications and copy-number variation in the human genome. Am J Hum Genet. (2005) 77:78-88. doi: 10.1086/431652

45. Jun J, Ryvkin P, Hemphill E. Duplication mechanism and disruptions in flanking regions determine the fate of Mammalian gene duplicates. J Comput Biol. (2009) 16:1253-66. doi: 10.1089/cmb.2009.0074

46. Jault C, Pichon L, Cluba J. Toll-like receptor gene family and TIRdomain adapters in Danio rerio. Mol Immunol. (2004) 40:759-71. doi: 10.1016/j.molimm.2003.10.001

47. Meijer AH, Gabby Krens SF, Medina Rodriguez IA, He S, Bitter W, SnaarJagalska BE, et al. Expression analysis of the Toll-like receptor and TIR domain adaptor families of zebrafish. Mol Immunol. (2004) 40:773-83. doi: 10.1016/j.molimm.2003.10.003

48. Palti Y, Gahr SA, Purcell MK, Hadidi S, Rexroad III CE, Wiens GD. Identification, characterization and genetic mapping of TLR7, TLR8al and TLR8a2 genes in rainbow trout (Oncorhynchus mykiss). Dev Comp Immunol. (2010) 34:219-33. doi: 10.1016/j.dci.2009.10.002

49. Kongchum P, Hallerman EM, Hulata G, David L, Palti Y. Molecular cloning, characterization and expression analysis of TLR9, MyD88 and TRAF6 genes in common carp (Cyprinus carpio). Fish Shellfish Immun. (2011) 30:361-71. doi: $10.1016 /$ j.fsi.2010.11.012

50. Kondrashov FA. Gene duplication as a mechanism of genomic adaptation to a changing environment. Proc R Soc B Biol Sci. (2012) 279:5048-57. doi: $10.1098 /$ rspb.2012.1108

51. Sun $\mathrm{M}$, Mu Y, Ding Y, Ao J, Chen X. Molecular and functional characterization of Toll-like receptor 21 in large yellow croaker (Larimichthys crocea). Fish Shellfish Immun. (2016) 59:179-88. doi: 10.1016/j.fsi.2016. 10.024

52. Lee PT, Zou J, Holland JW, Martin SA, Collet B, Kanellos T, et al. Identification and characterisation of TLR18-21 genes in Atlantic salmon (Salmo salar). Helminthologia. (2014) 41:549-59. doi: 10.1016/j.fsi.2014.10.006

53. Keestra AM, de Zoete MR, Bouwman LI, van Putten JP. Chicken TLR21 is an innate CpG DNA receptor distinct from mammalian TLR9. J Immunol. (2010) 185:460-7. doi: 10.4049/jimmunol.0901921

54. Yeh DW, Liu YL, Lo YC, Yuh CH, Yu GY, Lo JF, et al. Toll-like receptor 9 and 21 have different ligand recognition profiles and cooperatively mediate 
activity of CpG-oligodeoxynucleotides in zebrafish. Proc Natl Acad Sci USA. (2013) 110:20711-6. doi: 10.1073/pnas.1305273110

55. Lu YC, Yeh WC, Ohashi PS. LPS/TLR4 signal transduction pathway. Cytokine. (2008) 42:145-51. doi: 10.1016/j.cyto.2008.01.006

56. Sepulcre MP, Alcaraz-Pérez F, López-Muñoz A, Roca FJ, Meseguer J, Cayuela ML, et al. Evolution of lipopolysaccharide (LPS) recognition and signaling: fish TLR4 does not recognize LPS and negatively regulates NF-KB activation. J Immunol. (2009) 182:1836-45. doi: 10.4049/jimmunol.0801755

57. Novoa B, Bowman T, Zon L, Figueras A. LPS response and tolerance in the zebrafish (Danio rerio). Fish Shellfish Immun. (2009) 26:326-31. doi: 10.1016/j.fsi.2008.12.004

58. Swain P, Nayak SK, Nanda PK, Dash S. Biological effects of bacterial lipopolysaccharide (endotoxin) in fish: a review. Fish Shellfish Immun. (2008) 25:191-201. doi: 10.1016/j.fsi.2008.04.009

59. Bi D, Wang Y, Gao Y, Li X, Chu Q, Cui J, et al. Recognition of lipopolysaccharide and activation of NF- $\mathrm{KB}$ by cytosolic sensor NOD1 in teleost fish. Front Immunol. (2018) 9:1413. doi: 10.3389/fimmu.2018.01413

60. Lu X, Ning Y, Liu H, Nie L, Chen J. A Novel lipopolysaccharide recognition mechanism mediated by internalization in teleost macrophages. Front Immunol. (2018) 9:2758. doi: 10.3389/fimmu.2018.02758

61. Hurst LD, Pál C, Lercher MJ. The evolutionary dynamics of eukaryotic gene order. Nat Rev Genet. (2004) 5:299-310. doi: 10.1038/nrg1319

62. Young RM, Staudt LM. Targeting pathological B cell receptor signaling in lymphoid malignancies. Nat Rev Drug Discov. (2013) 12:229-43. doi: $10.1038 / \mathrm{nrd} 3937$

63. Hemmi H, Takeuchi O, Kawai $T$, Kaisho T, Sato S, Sanjo H, et al. A toll-like receptor recognizes bacterial DNA. Nature. (2000) 408:740-5. doi: $10.1038 / 35047123$

64. Federspiel JD, Codreanu SG, Palubinsky AM, Winland AJ, Betanzos CM, McLaughlin B, et al. Assembly Dynamics and Stoichiometry of the Apoptosis Signal-regulating Kinase (ASK) signalosome in response to electrophile stress. Mol Cell Proteomics. (2016) 15:1947-61. doi: 10.1074/mcp.M115.057364

65. Matsuzawa A, Saegusa, K, Noguchi T, Sadamitsu C, Nishitoh H, Nagai S, et al. ROS-dependent activation of the TRAF6-ASK1-p38 pathway is selectively required for TLR4-mediated innate immunity. Nat Immunol. (2005) 6:587-92. doi: $10.1038 /$ ni1200

66. Xiao X, Qin Q, Chen X. Molecular characterization of a Toll-like receptor 22 homologue in large yellow croaker (Pseudosciaena crocea) and promoter activity analysis of its 5'-flanking sequence. Fish Shellfish Immun. (2011) 30:224-33. doi: 10.1016/j.fsi.2010.10.014

67. Hu GB, Zhang SF, Yang X, Liu DH, Liu QM, Zhang SC. Cloning and expression analysis of a Toll-like receptor 22 (tlr22) gene from turbot, Scophthalmus maximus. Fish Shellfish Immun. (2015) 44:399-409. doi: 10.1016/j.fsi.2015.03.001

68. Matsuo A, Oshiumi H, Tsujita T, Mitani H, Kasai H, Yoshimizu $\mathrm{M}$, et al. Teleost TLR22 recognizes RNA duplex to induce IFN and protect cells from birnaviruses. J Immunol. (2008) 181:3474-85. doi: 10.4049/jimmunol.181.5.3474

69. Shi Q, Xiong B, Zhong J, Wang H, Ma D, Miao C. MFHAS1 suppresses TLR4 signaling pathway via induction of PP2A C subunit cytoplasm translocation and inhibition of c-Jun dephosphorylation at Thr239. Mol Immunol. (2017) 88:79-88. doi: 10.1016/j.molimm.2017.06.017

70. Yao XQ, Li XC, Zhang XX, Yin YY, Liu B, Luo DJ, et al. Glycogen synthase kinase- $3 \beta$ regulates leucine-309 demethylation of protein phosphatase-2A via PPMT1 and PME-1. Febs Lett. (2012) 586:2522-8. doi: 10.1016/j.febslet.2012.06.018

71. Andreassen R, Høyheim B. miRNAs associated with immune response in teleost fish. Dev Comp Immunol. (2017) 75:77-85. doi: 10.1016/j.dci.2017.02.023

72. Zhou Z, Lin Z, Pang X, Shan P, Wang J. MicroRNA regulation of Tolllike receptor signaling pathways in teleost fish. Fish Shellfish Immun. (2018) 75:32-40. doi: 10.1016/j.fsi.2018.01.036

73. Souvik M, Neeta SR, Wagener DK, Majumder PP. Signatures of natural selection are not uniform across genes of innate immune system, but purifying selection is the dominant signature. Proc Natl Acad Sci USA. (2009) 106:70738. doi: 10.1073/pnas.0811357106

74. Dirk W, Jann OC, Victoria O, Glass EJ, Coffey TJ. Variation matters: TLR structure and species-specific pathogen recognition. Trends Immunol. (2009) 30:124-30. doi: 10.1016/j.it.2008.12.001

75. Bell JK, Mullen GED, Leifer CA, Alessandra M, Davies DR, Segal DM. Leucine-rich repeats and pathogen recognition in Toll-like receptors. Trends Immunol. (2003) 24:528-33. doi: 10.1016/S1471-4906(03)00242-4

Conflict of Interest Statement: The authors declare that the research was conducted in the absence of any commercial or financial relationships that could be construed as a potential conflict of interest.

Copyright (c) 2019 Qiu, Fernandes, Hong, Wu, Zhang, Huang, Liu, Yu, Wang, You and Chen. This is an open-access article distributed under the terms of the Creative Commons Attribution License (CC BY). The use, distribution or reproduction in other forums is permitted, provided the original author(s) and the copyright owner(s) are credited and that the original publication in this journal is cited, in accordance with accepted academic practice. No use, distribution or reproduction is permitted which does not comply with these terms. 